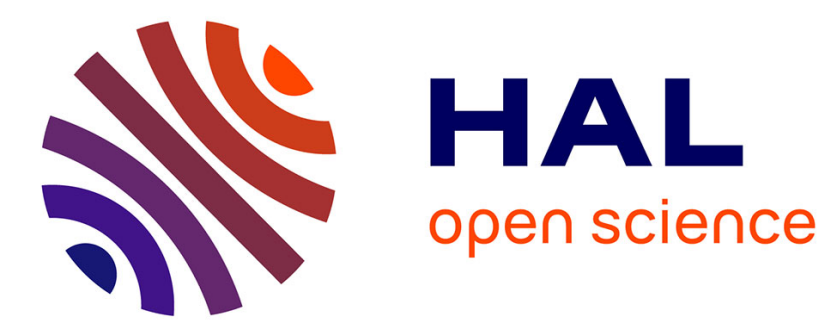

\title{
Experimental investigation on the grain-scale compression behavior of loose wet granular material
}

Vinh Du Than, Patrick Aimedieu, Jean-Michel Pereira, Jean-Noël Roux, Anh Minh A.M. Tang

\section{- To cite this version:}

Vinh Du Than, Patrick Aimedieu, Jean-Michel Pereira, Jean-Noël Roux, Anh Minh A.M. Tang. Experimental investigation on the grain-scale compression behavior of loose wet granular material. Acta Geotechnica, 2020, 15 (1039-1055), 10.1007/s11440-019-00856-0 . hal-02879312

HAL Id: hal-02879312

https://hal-enpc.archives-ouvertes.fr/hal-02879312

Submitted on 23 Jun 2020

HAL is a multi-disciplinary open access archive for the deposit and dissemination of scientific research documents, whether they are published or not. The documents may come from teaching and research institutions in France or abroad, or from public or private research centers.
L'archive ouverte pluridisciplinaire $\mathbf{H A L}$, est destinée au dépôt et à la diffusion de documents scientifiques de niveau recherche, publiés ou non, émanant des établissements d'enseignement et de recherche français ou étrangers, des laboratoires publics ou privés. 


\section{Experimental investigation on the grain-scale compression}

\section{2 behavior of loose wet granular material}

3

4 Vinh-Du Than ${ }^{1,2}$, Patrick Aimedieu ${ }^{1}$, Jean-Michel Pereira ${ }^{1}$, Jean-Noël Roux ${ }^{1}$, Anh Minh $5 \quad$ Tang $^{1}$

6

$7{ }^{1}$ Université Paris-Est, Laboratoire Navier, UMR 8205, École des Ponts ParisTech, IFSTTAR, 8 CNRS, 77455 Marne-la-Vallée Cedex 2, France

$9{ }^{2}$ The University of Danang, University of Technology and Education, Department of Civil

10 Engineering, Danang, Vietnam

11

12

13 Corresponding author:

14

Dr. Anh Minh TANG

16

17 Ecole des Ponts ParisTech

18 6-8 avenue Blaise Pascal

1977455 MARNE-LA-VALLEE

20 France

21 Tel: $+33.1 \cdot 64 \cdot 15.35 .63$

22 Email: anhminh.tang@enpc.fr 
30 Abstract

31

32 The behavior of model granular materials (glass beads) wetted by a small quantity of liquid

33 forming capillary bridges is studied by one-dimensional compression test combined with $\mathrm{X}$ -

34 ray computed tomography (XRCT) observation. Special attention is paid to obtain very loose

35 initial states (initial void ratio of about 2.30) stabilized by capillary cohesion. XRCT-based

36 analyses involve spherical particle detection adapted to relatively low-resolution images,

37 which enable heterogeneities to be visualized and microstructural information to be collected.

38 This study on an ideal material provides an insight into the macroscopic compression

39 behavior of wet granular materials based on the microstructural change, such as pore distance

40 distribution, coordination number of contacts, coordination number of neighbors, number of

41 contacts per grain.

42

43 Keywords: Wet granular material; Microstructure; One-dimensional compression; X-ray

44 computed tomography; Grain-scale analysis

45

46

47

48

49

50

51

52

53

54

55 
$57 \quad$ List of notation

58

$59 a \quad$ size of standard volume

$60 \quad b \quad$ size of extended volume

$61<d>\quad$ average diameter

$62 d_{\min } \quad$ minimum diameter

$63 d_{\max } \quad$ maximum diameter

$64 e_{0} \quad$ initial void ratio

$65 e \quad$ void ratio

66 EV $\quad$ extended volume

$67 \quad f(r) \quad$ signature curve

$68 \Phi_{0} \quad$ initial solid fraction

$69 \quad g(r) \quad$ radial distribution function

$70 \quad i, j, k \quad$ voxel indices

$71 i_{\mathrm{C}}, j_{\mathrm{C}}, k_{\mathrm{C}} \quad$ center position of detected sphere

$72 \mathbf{I}(i, j, k) \quad$ intensity at voxel $(i, j, k)$

$73 \nabla \mathbf{I}(i, j, k) \quad$ gradient vector at voxel $(i, j, k)$

$74 N \quad$ number of particles

$75 \quad N_{\mathrm{C}} \quad$ number of pairs in contacts

$76 p(r) \quad$ average number density of particles

$77 q(i, j, k) \quad$ vector from $\left(i_{\mathrm{C}}, j_{\mathrm{C}}, k_{\mathrm{C}}\right)$ to voxel $(i, j, k)$

$78 r \quad$ radii of particles / radial distance

79 SV $\quad$ standard volume

$80 \quad \mathrm{Si} \quad$ step of scan (S1, S2, S3, S4)

$81 z \quad$ total coordination number 


\section{Introduction}

85 Granular materials appear in various natural and industrial processes. They are often composed of individual grains that vary in shape, size and surface texture. Such parameters can remarkably affect the packing of granulates and their contact distribution characteristics.

In the case of wet granular materials, the existence of liquid menisci between particles plays a key role in the overall behavior of the assembly. Capillary cohesion bestows to these materials

90 specific mechanical features that do not exist with dry grains, such as the ability to form stable structures with very low densities, and a strong sensitivity to external applied forces. So far, there are a lot of experimental studies on their macro- and micro-mechanical behaviors, such as: cohesive soils $[1,2]$, wet beads $[3,4]$, loess [5, 6], wet sand [7-9], among others. These works evidenced the link between the macroscopic mechanical behavior and the microstructure change. However, few works have yet focused on the grain-scale behavior.

97 Currently, the mechanical grain-scale behavior of a wet granular material is usually investigated by discrete element methods using 2D and/or 3D simulations [10-24]. These studies precisely characterized the change of microstructure such as coordination numbers (of

100 contacts, of distant interactions, of compressive and tensile contacts), radial distribution

101 function, force chains, distribution of forces between particles, etc. under the applied external forces. However, few works have compared numerical simulations (e.g. using the discrete element method) and experimental results in order to validate the numerical method [25-30].

105 X-ray computed tomography (XRCT) has tremendous potential due to its ability to carry out 106 volumetric imaging inside the specimen. It has become a widely used technique and is 107 appropriate for numerous types of materials including particulate and porous materials [31, 
108 32]. Furthermore, this technique has been used in several studies to investigate the

109 microstructure of granular materials under mechanical loading [8, 33-39]. Most of these

110 works focus on the analysis of granular packing in dense states and with large-size

111 particulates. Few works have experimentally investigated the mechanical grain-scale behavior

112 of wet granular materials by using XRCT [40-44].

113

114 In this paper, we present an experimental investigation, by means of XRCT, on a loose wet

115 granular material. A cylindrical sample constituted of thousands of glass beads with liquid

116 bonds were subjected to an oedometric compression test, which consisted of several

117 compression steps. At each step, a XRCT scan was performed. The sample heterogeneity is

118 first analyzed in order to get a general understanding of the pore-scale compression behavior.

119 The main objective of the present works is to give a further insight into the grain-scale

120 compression behavior of loose wet granular materials.

\section{Experiments}

\subsection{Material and specimen preparation}

125 The investigated material consists of industrial spherical glass beads (diameter equals $87 \mu \mathrm{m}$

126 to $110 \mu \mathrm{m}$ with an average value of $100 \mu \mathrm{m})$. The specific density of this material is equal to

$1272,460 \mathrm{~kg} \cdot \mathrm{m}^{-3}$. A Scanning Electronic Microscopic image of a glass bead is shown in Figure 1.

128 The image shows that the surface of the bead is slightly damaged (beside the presence of 129 some tiny dust grains) which makes it not perfectly smooth.

131 To prepare the specimen, the glass beads were first mixed with water to reach gravimetric

132 water content (mass of water divided by mass of solid) of $3.5 \%$, which ensured that the 133 specimens always remained in the pendular regime. The wetted glass beads were then placed 
134 on a $200 \mu \mathrm{m}$ opening sieve installed $80 \mathrm{~mm}$ above an oedometric cell. Afterward, a machine

135 vibrated the sieve vertically in order to gradually pass the wet glass beads through the sieve 136 and let them fall into the cell.

137

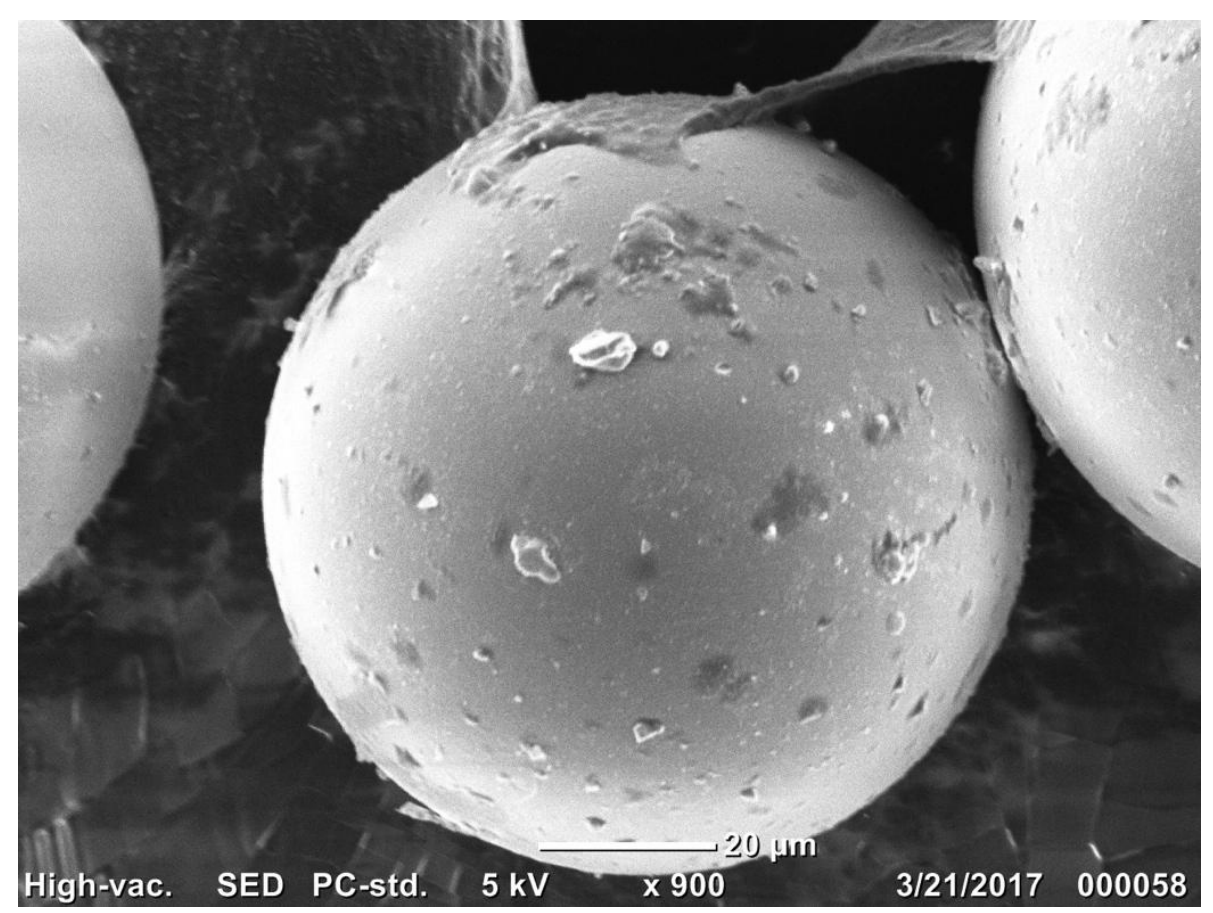

139 Figure 1: Scanning Electronic Microscopic image of a glass bead.

141 This procedure allowed the preparation of loose specimen with a porosity $n=0.70$

142 (corresponding to a void ratio $e_{0}=2.33$, a solid fraction $\Phi_{0}=0.30$, and a degree of saturation 143 of $S_{r}=3.7 \%$ ). This degree of saturation, smaller than $5 \%$, corresponds to the pendular regime 144 following Newitt \& Conway-Jones [45].

\subsection{Oedometric compression behavior}

148 In order to perform oedometric compression tests, the specimen (50 $\mathrm{mm}$ in diameter and 20

$149 \mathrm{~mm}$ in height), prepared by the procedure described above, was carefully placed on a loading 150 platform. A piston was fixed to a load cell to measure the vertical load applied to the sample. 
151 A LVDT sensor measured the displacement corresponding to the compression. The cell was

152 then moved upward with a rate of $0.5 \mathrm{~mm} / \mathrm{min}$.

153

154 Figure 2 illustrates the compression curves of two specimens (CT01 and CT02). Based on the

155 shape of these curves and the range of vertical stress, three stages could be identified. Firstly,

156 under low stresses $\left(\sigma_{\mathrm{v}}<0.1 \mathrm{kPa}\right)$, void ratio $e$ is slightly decreased. Secondly, the void ratio is

157 sharply decreased. Finally, under higher stresses $\left(\sigma_{\mathrm{v}}>10 \mathrm{kPa}\right)$, the void ratio is gradually

158 decreased.

159

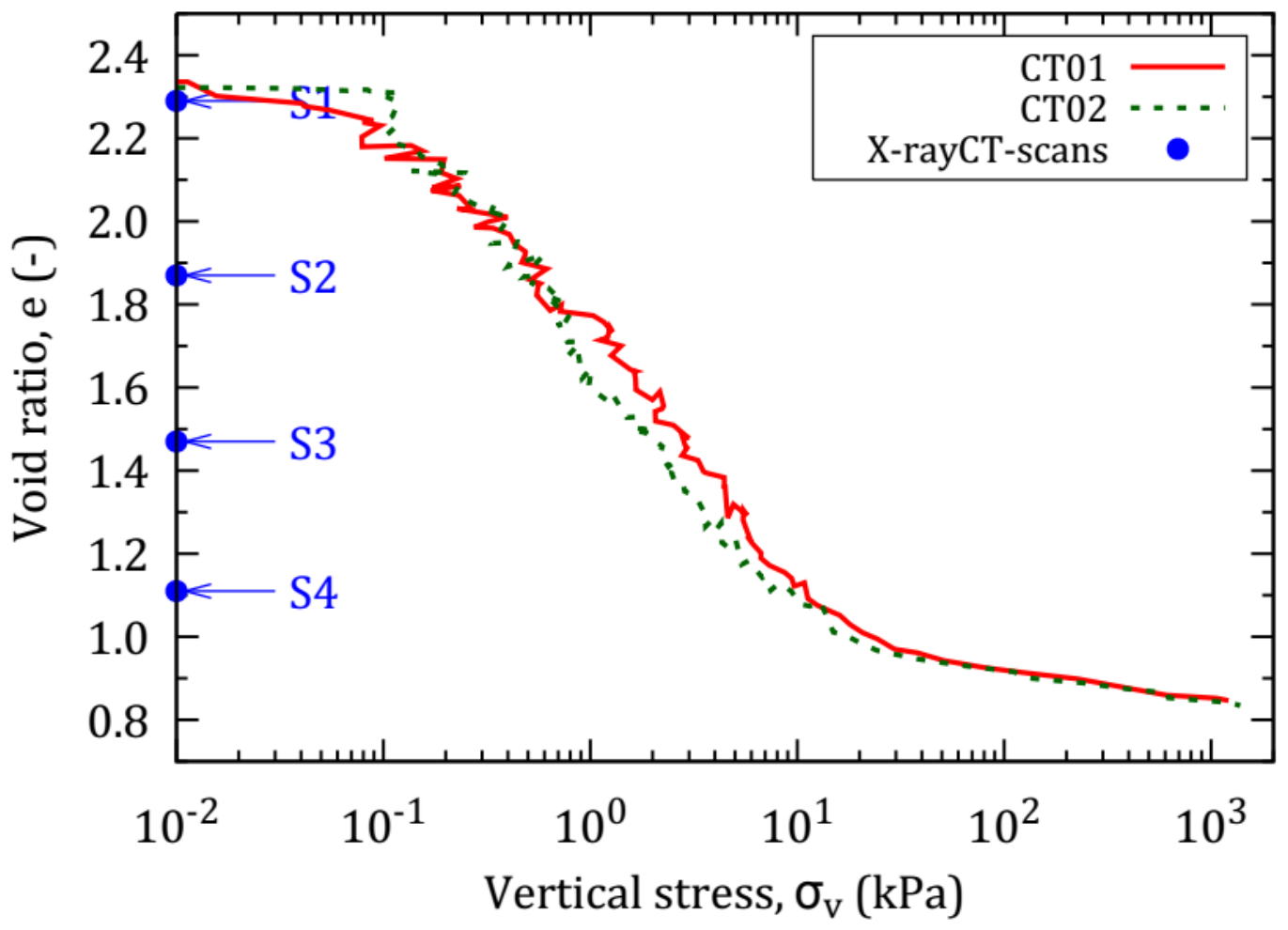

161 Figure 2: Void ratio versus vertical stress during oedometric compression tests for two specimens. The circle points show the conditions of XRCT scans.

163

164

\subsection{X-ray computed tomography}

165 
166 In the present work, XRCT scans were performed by using an UltraTom microtomography

167 device. Prior to the observations with XRCT, the specimen $(20 \mathrm{~mm}$ in diameter and $10 \mathrm{~mm}$ in

168 height), prepared by the procedure described in the section 2.1 , was compressed to reduce its 169 void ratio to $e=2.29$ (position S1, see Figure 2). Once this state was reached, a XRCT scan

170 (global scan) was performed for the whole specimen. The central zone of the sample (10 $\mathrm{mm}$

171 in diameter and $80 \%$ of the sample's height) was subsequently scanned (local scan). Figure 3

172 shows an example of reconstructed 3D images obtained from these scans. The voxel size was

$17313 \mu \mathrm{m}$ for the global scan and $6.5 \mu \mathrm{m}$ for the local scan. Following the scan corresponding to

174 the position S1, the same scanning procedure was repeated three times to obtain the XRCT

175 images corresponding to positions S2, S3 and S4, as shown in Figure 2. One piston having

176 appropriate length was designed for each position. That allowed the correct control of the

177 height of the sample to be scanned (there was no pressure applied to the piston during the

178 scan). A global scan took $80 \mathrm{~min}$ and a local scan took four hours. During these scans, the

179 piston was maintained above the sample to avoid evaporation of water. The small gap

180 between the piston and the cell $(0.05 \mathrm{~mm})$ allows draining air during compression while

181 avoiding evaporation during the scan. The cell and the pistons were made of Polymethyl

182 methacrylate (PMMA) in order to optimize the quality of the XRCT images.

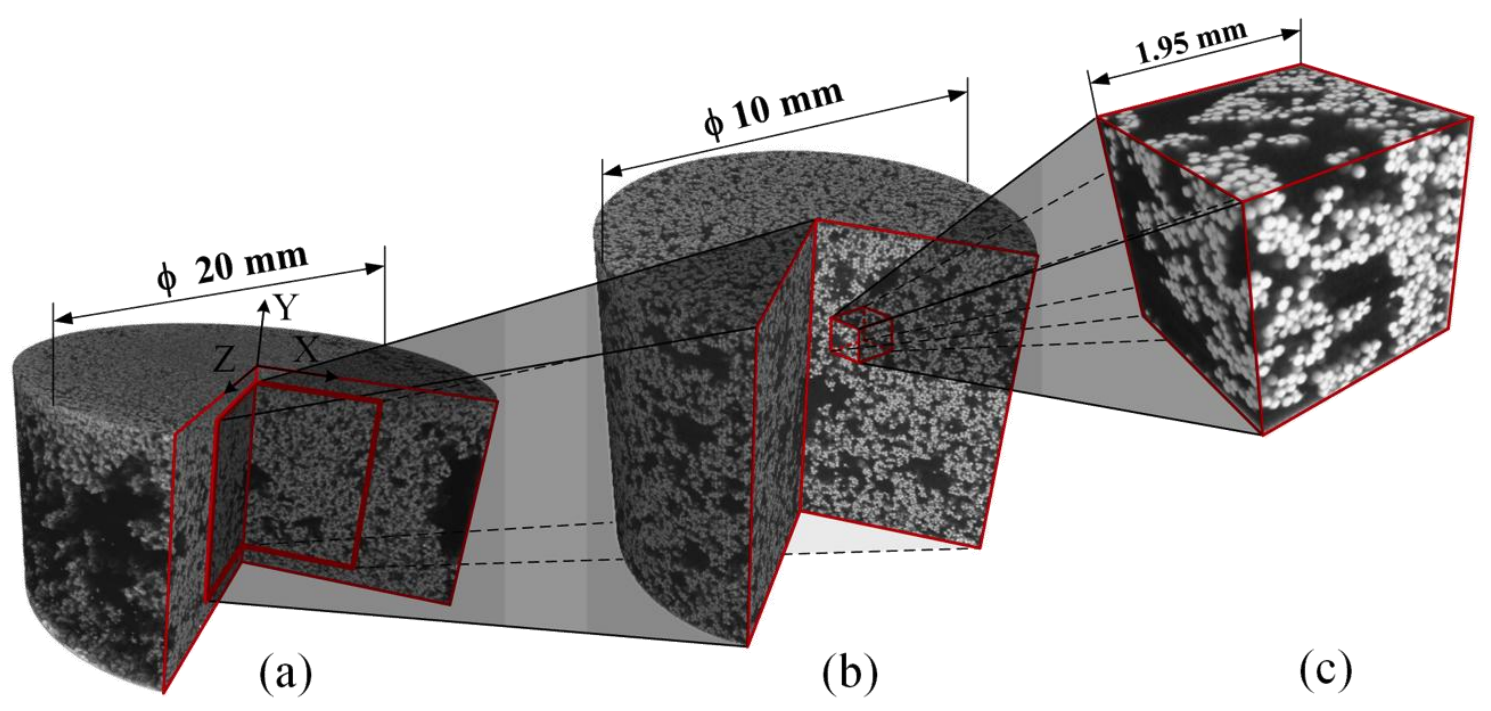


Figure 3: (a) Global scan, (b) local scan, and (c) investigated cube.

186

\subsection{Analysis of sample heterogeneity}

188

189 The reconstructed 3D samples, obtained by global scans at four compaction levels, are shown

190 in Figure 4. It is well recognized that the density is not homogeneous within the cylindrical

191 sample, especially at the periphery where more voids can be observed; higher densities and

192 fewer voids exist at the top and the bottom of the sample. There are also several large voids in

193 the center of the specimen in the initial stages (Figure 4ab). Similar results were observed

194 previously on unsaturated loose volcanic sands [44]. This heterogeneity is due to the

195 specimen preparation, and also to the boundary conditions imposed by the oedometer cell. In

196 other words, during the specimen preparation, the friction between the cell and the grains

197 limits the movement of grains. At higher compaction levels (Figure $4 c d$ ), the structure is

198 strongly rearranged in the central layers because the loosest state was observed in this region

199 (horizontal band) while the structure is slightly affected in the bottom and top layers.

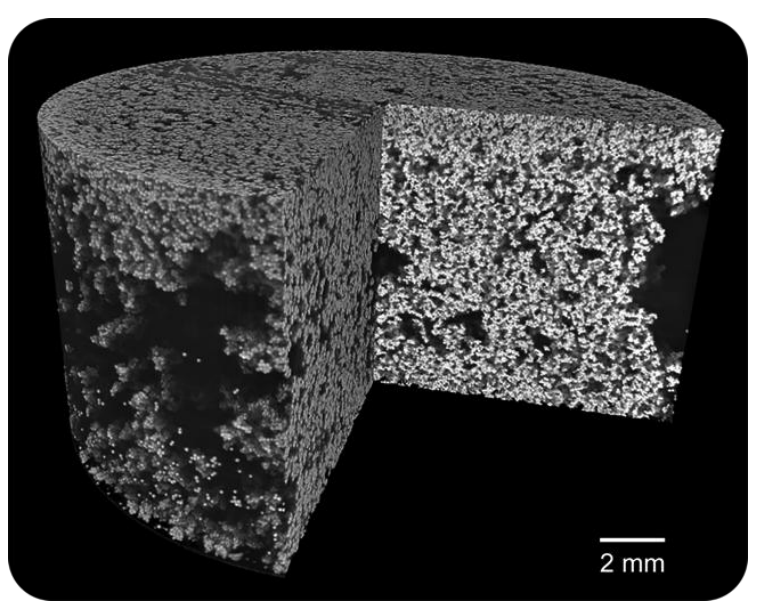

(a)

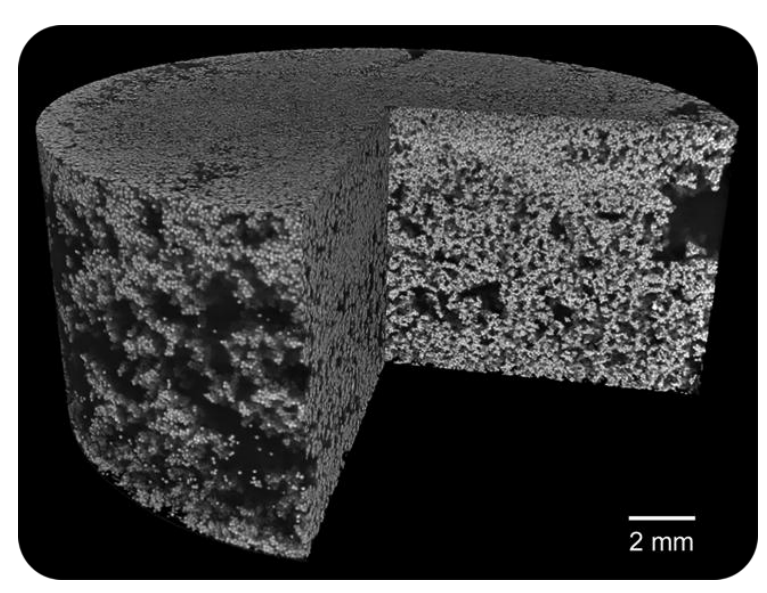

(b) 


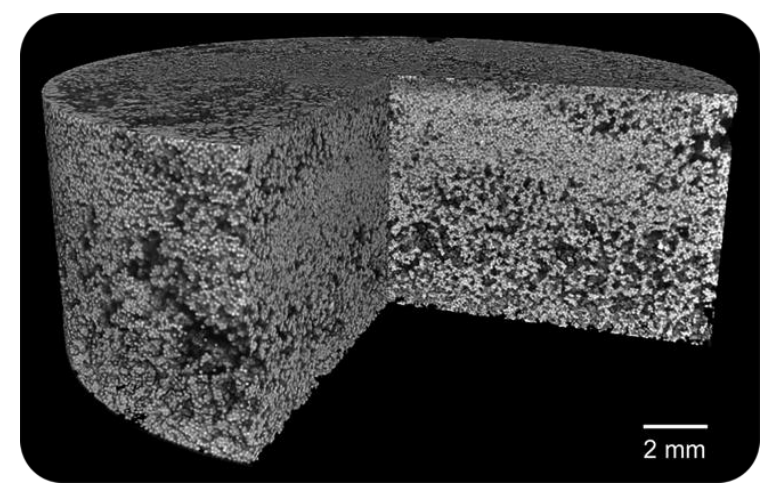

(c)

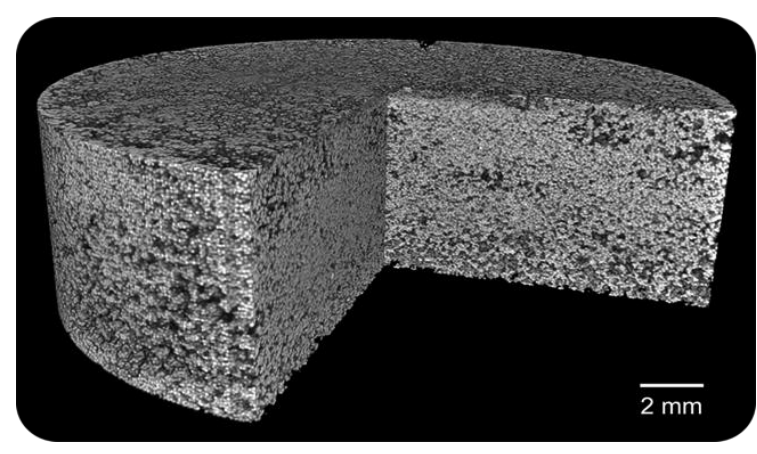

(d)

201 Figure 4: 3D reconstructed images of the sample at different compaction levels. (a) S1, (b) S2,

202 (c) S3, and (d) S4.

203

204 The grey level histograms are shown in Figure 5. Depending on the compaction level, two

205 peaks are more or less visible on each histogram. The first one on the left-hand side

206 corresponds to air-dominated voxels. Its number is smaller at a denser state. The peak on the

207 right-hand side corresponds to grain-dominated voxels and its number did not change during 208 compaction.

209

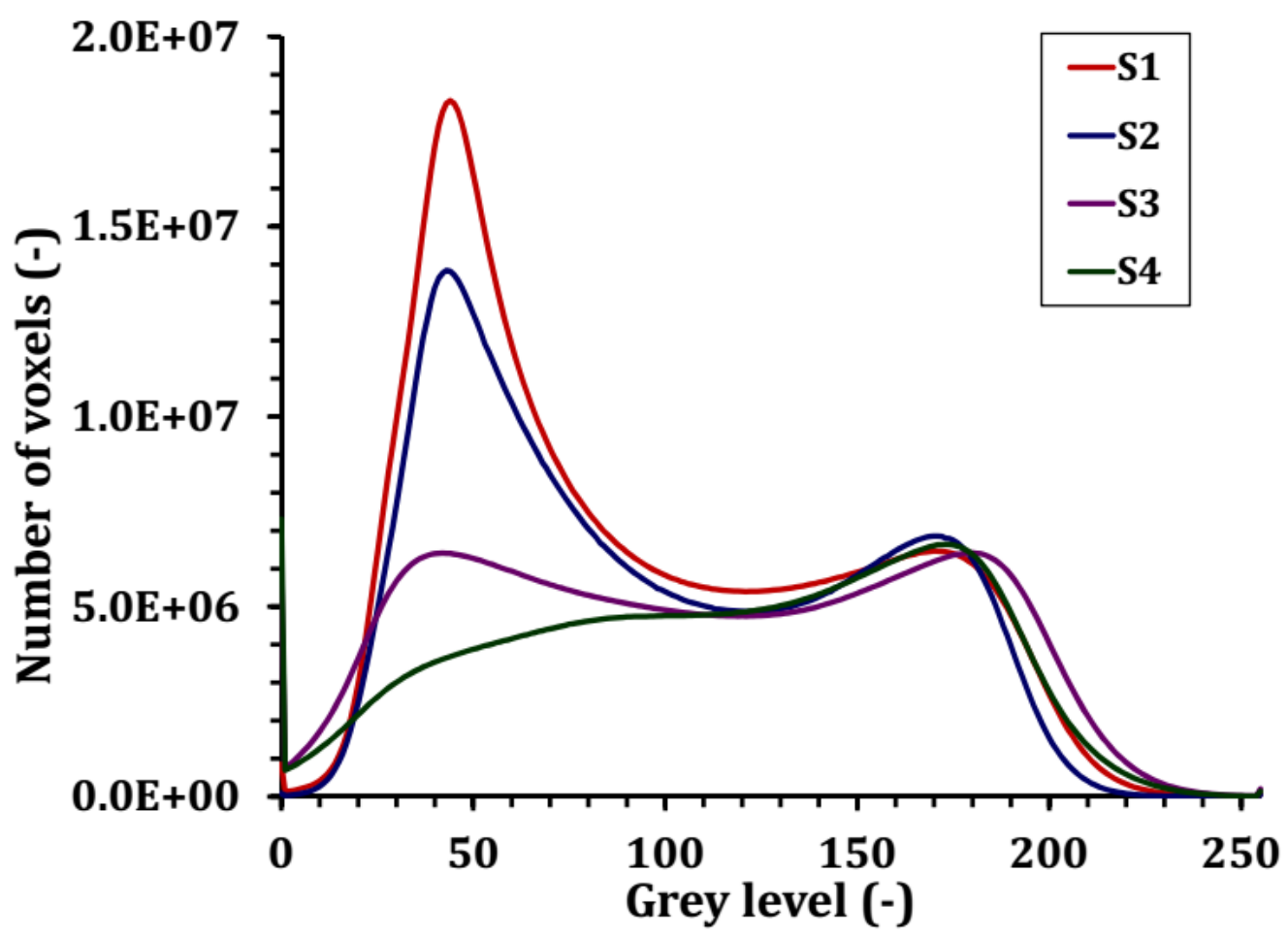


211 Figure 5. Grey level histogram for the four scans.

212

213 The void ratio distribution according to the elevation $(h)$ during the compression is illustrated

214 in Figure 6. The void ratio is directly calculated from the tomography images. To obtain void

215 ratio values, a grey-level threshold value was chosen for each scan, based on their grey level

216 histogram. This value (around 130) was determined within the interval between the peaks

217 corresponding to the air phase and the aggregates, respectively. The IsoData method [46] was

218 chosen for the segmentation process. After thresholding, the aggregates (white) and the air-

219 filled voids (black) are clearly distinguished. The void ratio is then recalculated by dividing

220 the number of black voxels by the number of white voxels of the segmented image. Note that

221 for the four global scans, the void ratios obtained by this method are similar to the imposed

222 values; the average void ratios of the four curves $(2.31,1.91,1.48$ and 1.14 , corresponding to

$223 \mathrm{~S} 1, \mathrm{~S} 2, \mathrm{~S} 3$ and S4) are very close to the macroscopic ones, which are 2.29, 1.87, 1.47, and

2241.11 respectively. In this study, as the volume of water is smaller than $5 \%$ of volume of voids

225 at the beginning, the presence of water has been ignored during the whole image analysis

226 process. The binarization and watershed approach are not used because of the low resolution

227 of the images. The voxel size was $13 \mu \mathrm{m}$, for the global scan, that means each grain has

228 around 7 pixels diameter. In other words, the contact boundary of grains is not clear to

229 separate. In fact, we had tried to apply the sub-voxel algorithm in order to increase the

230 resolution of image, especially at the contact boundary but this method increases significantly

231 the computation time. 


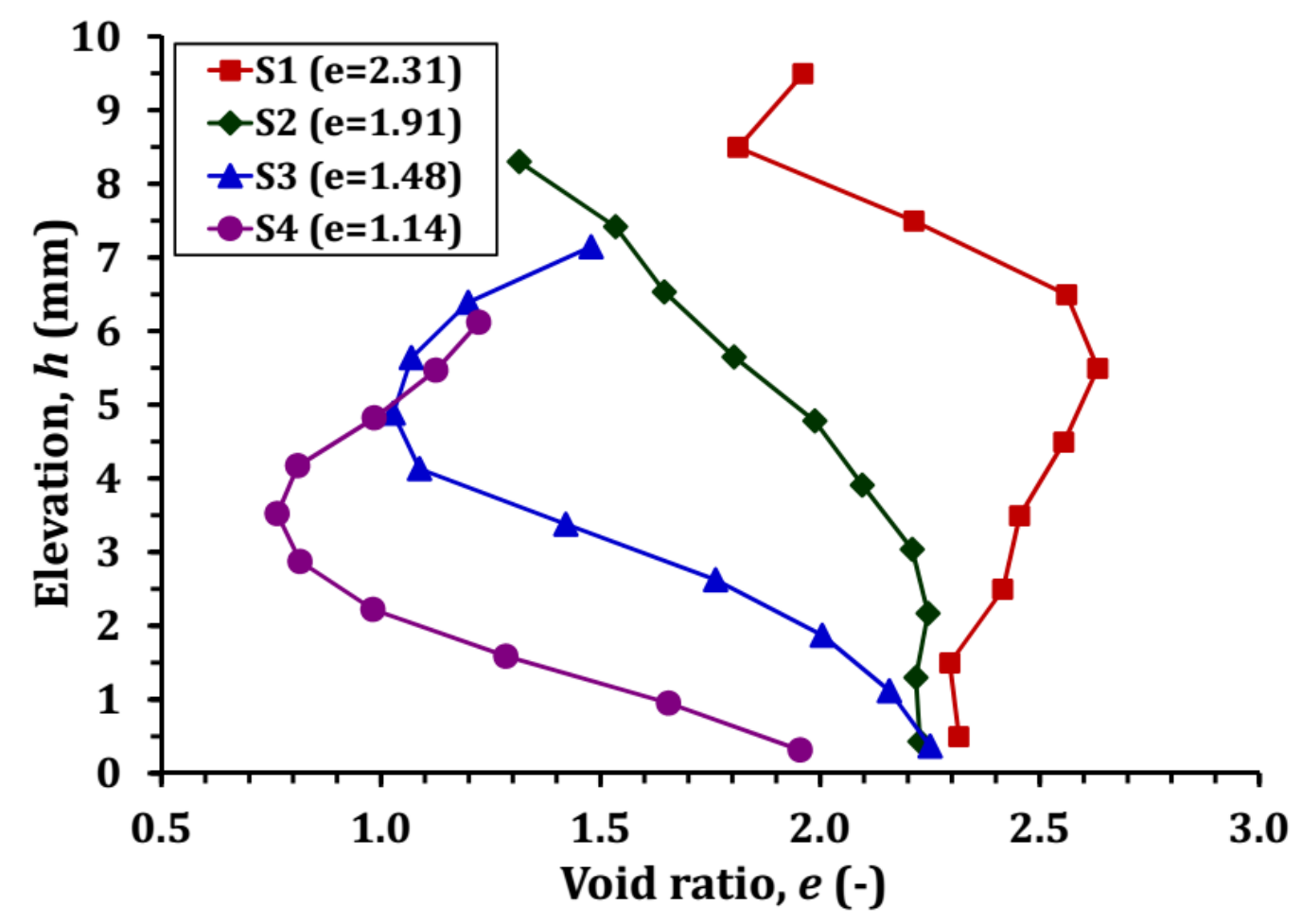

Figure 6: Void ratio versus elevation at different compaction levels. Note that the value in the parenthesis is the average void ratio.

In the initial state $\left(\mathrm{S} 1-\right.$ red curve), the void ratio is largest at $h=5.5 \mathrm{~mm}\left(e_{\max } \approx 2.65\right)$

238 because of the existence of several large pores in the center and the periphery of the specimen 239 (see Figure 4a). Meanwhile the sample is denser at the bottom and densest at the top. The 240 higher density at the bottom could be explained by a higher stress induced by the weight of 241 the sample. The higher density at the top could be related to the preparation of the sample surface. Actually, after having filled the cell with wet grains, the excess grains on the top of 243 the sample were removed to obtain a planar sample top surface. Even if this action was done 244 with care, that would induce supplementary stress that slightly compacted the top layer of the 245 sample. At the stage S2 (dark-green curve), the structure is notably compacted and 246 rearranged; void ratio in the middle and at the top is significantly reduced. The structure in the 247 middle is remarkably disturbed when passing the stage S2 to S3 (blue curve). At the densest 248 state (S4 - dark-purple curve), a strong decrease of void ratio is obtained in the middle $\left(e_{\min }=\right.$ 249 0.7) while the structure at the bottom is slightly rearranged. 
2513 Processing for local scan images

252

253

254

\subsection{Principles}

Based on the local scans, virtual cubical specimens (edge length $=1.95 \mathrm{~mm}$ ) were extracted to analyze the grain-scale behavior of the sample during compression. Hereafter, these cubes are referred to as Standard Volume, or SV; one example is shown above in Figure $3 c$. Ten SVs were extracted at ten different positions (at the top, at the bottom, and in the central layers; and for each layer, at the periphery and at the center) in order to consider the sample microstructure heterogeneity. The subsets did not spatially overlap each other. It is worth to note that a much higher number of SV, extracted randomly, would be preferable but it would significantly increase the computational cost.

The algorithm used to detect spherical structures within 3D images, initially developed by Xie et al. [47] for 3D images, was used and modified following Peng et al. [48] (proposed for 2D images) along with the Adaptive Hough Transform algorithm of Illingworth \& Kittler [49]. Note that this algorithm is not applicable for natural materials (i.e. sand grains) where more advanced methods are required $[50,51]$

\subsection{Algorithm for detecting spherical grains}

The algorithm to detect spherical structures is illustrated in Figure 7. A 3D image with a cubic shape, and formatted as a 3D array, is first extracted from the local scans. The position of the center of the spheres is then directly located within the 3D array. Afterward, the radii of the spheres (with identified central position) are identified. Finally, the 3D reconstruction process and analysis are carried out based on the detected spheres. 
277 The detailed description of the main algorithm (see dashed-red square in Figure 7) is given in

278 the following subsections.

279

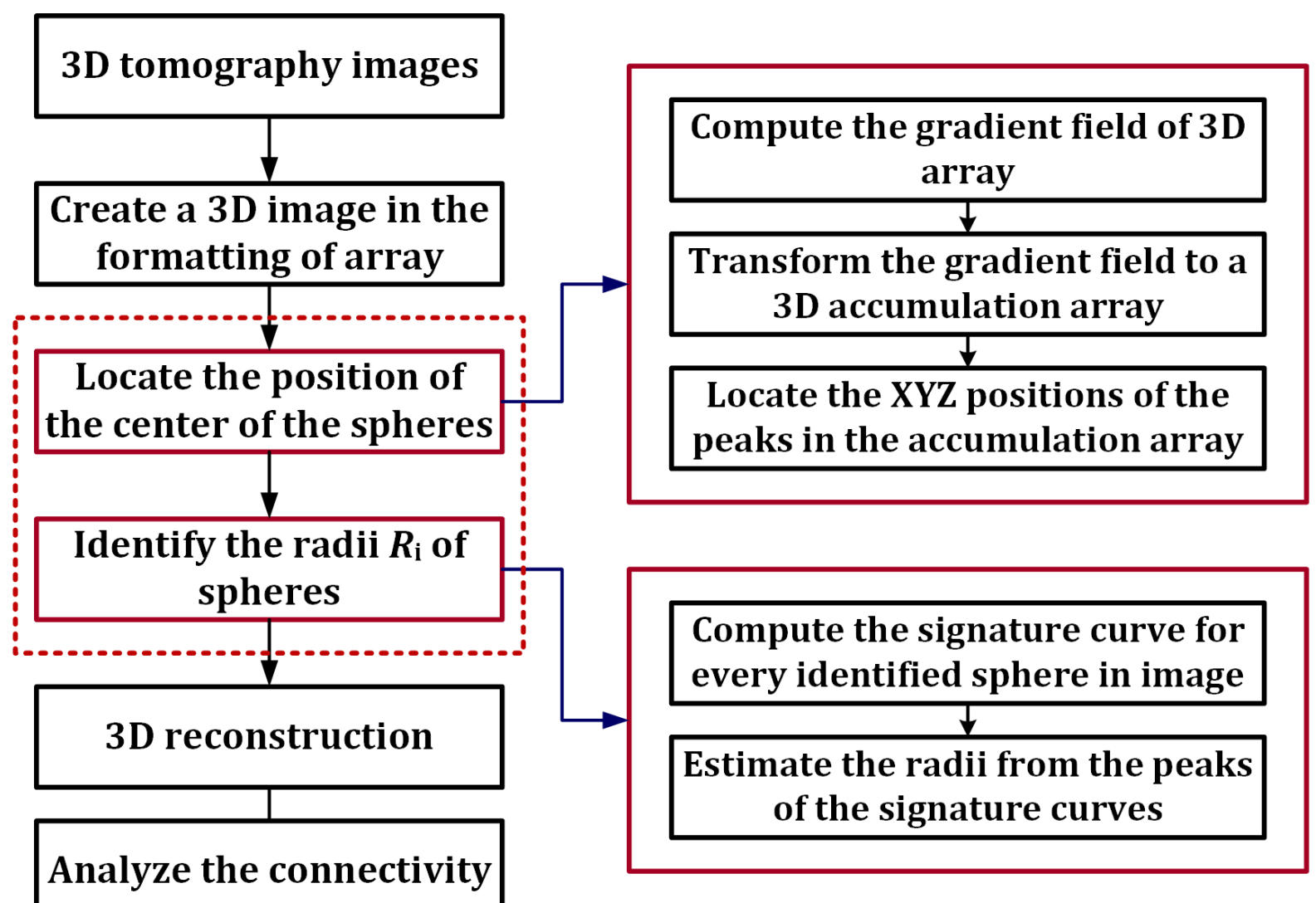

Figure 7: Algorithm used to detect the spherical structures.

\subsubsection{Locating the center of the spheres}

To compute the center's position of a sphere in the 3D image, the voxels that belong to that

$\nabla \mathbf{I}(\boldsymbol{i}, \boldsymbol{j}, \boldsymbol{k})=\left.\left(\boldsymbol{V}_{x}, \boldsymbol{V}_{y}, \boldsymbol{V}_{z}\right)\right|_{(i, j, k)}$

$$
=(I(i, j, k)-I(i, j, k-1), I(i, j, k)-I(i, j-1, k), I(i, j, k)-I(i-1, j, k)),
$$


where $(i, j, k)$ are the voxel indices, $\nabla \mathbf{I}(i, j, k)$ is the gradient vector at voxel $(i, j, k)$, which consists of $x, y$ and $z$ components, and $\mathbf{I}(i, j, k)$ is the image intensity at voxel $(i, j, k)$.

294 In a gradient field, the nonzero gradient vectors are either pointing toward the center of a 295 sphere or away from it. So, a transform is defined to convert the gradient field to an 296 accumulation array, in which the voxel intensity corresponds to the probability of that voxel 297 being the center of a sphere. The accumulation array, constructed by an additive process (see 298 Figure 8), has the same dimension of the gradient field. In the accumulation image, the 299 maximum intensity represents the center position of a sphere. In the gradient field, a weight 300 value is added to the voxels which have a nonzero gradient vector $\nabla \mathbf{I}(i, j, k)$ in the 301 accumulation array. The line segment in each direction is defined by the vector $\nabla \mathbf{I}(i, j, k)$. The 302 length of the line segment is set to be the possible maximum diameter of the spheres, and the magnitude of $\nabla \mathbf{I}(i, j, k)$ is used as the weight value. The accumulation array is built from the collecting of all nonzero gradient vectors in all directions. In Figure 8, the darkest voxel has the maximum weight.
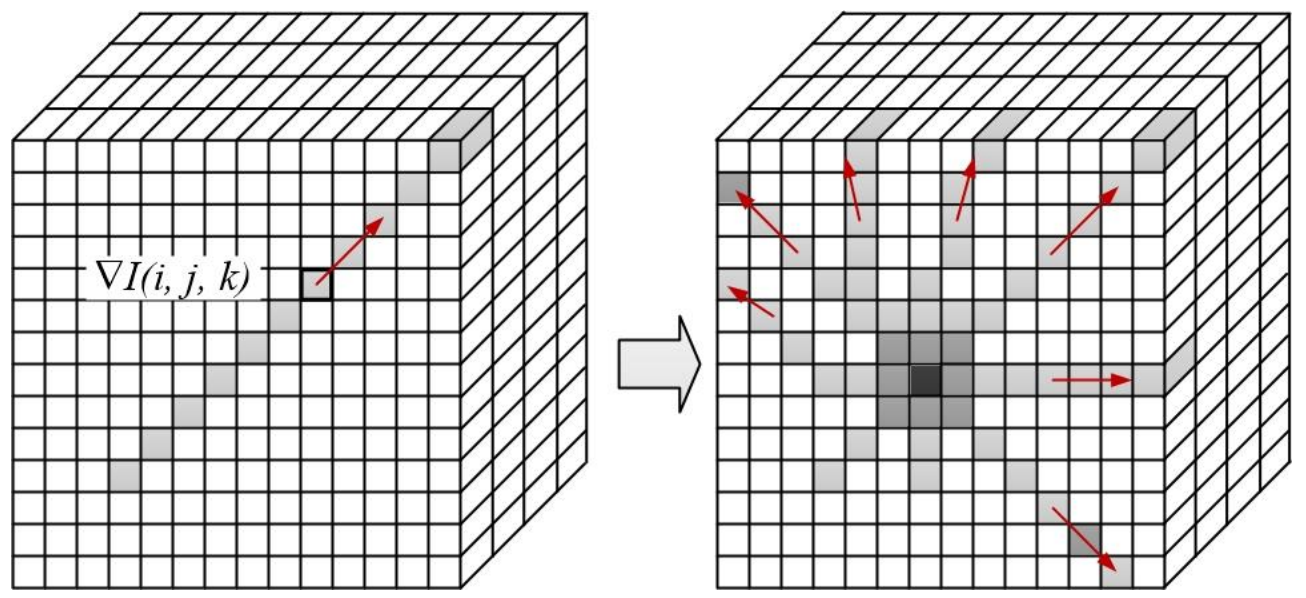

308 Figure 8: Construction of the accumulation array from the gradient field: the nonzero gradient 309 vector added to the accumulation array (left), and the accumulation array after adding the 310 nonzero gradient vectors in various directions (right). 
314 In order to determine the spheres' radius, a signature function $f(r)$ is defined over the gradient

315 field of the sphere's image, where $r$ is the distance to the center position of the sphere and $f(r)$

316 is the averaged dot product of the gradient vector and the radial vector.

318 Figure 9 shows the definition of the signature function, in which $\left(i_{\mathrm{c}}, j_{\mathrm{c}}, k_{\mathrm{c}}\right)$ is the center

319 position of a detected sphere. For a voxel $(i, j, k)$ in the neighborhood of $\left(i_{\mathrm{c}}, j_{\mathrm{c}}, k_{\mathrm{c}}\right), V(i, j, k)$ is

320 the gradient vector of $(i, j, k)$ and $q(i, j, k)$ is the vector joining $\left(i_{\mathrm{c}}, j_{\mathrm{c}}, k_{\mathrm{c}}\right)$ to the center of $(i, j$,

$321 k$ ). A sequence of discrete $r$ values with fixed interval is selected. It represents the distances to

322 the center of the sphere $\left(i_{\mathrm{c}}, j_{\mathrm{c}}, k_{\mathrm{c}}\right)$. For each $r$ value, $f(r)$ is defined by the following equation:

$$
f(r)=\frac{\sum_{|| \vec{q}(i, j, k)|-r|<\Delta r / 2}[\vec{V}(i, j, k) \cdot \vec{q}(i, j, k) /|\vec{q}(i, j, k)|]}{\sum_{|| \vec{q}(i, j, k)|-r|<\Delta r / 2} \mathbf{1}},
$$

\section{(Erreur ! Signet non défini.)}

324 where the sum is over all the voxels whose distance from $\left(i_{\mathrm{c}}, j_{\mathrm{c}}, k_{\mathrm{c}}\right)$ is within the range $[r$ -

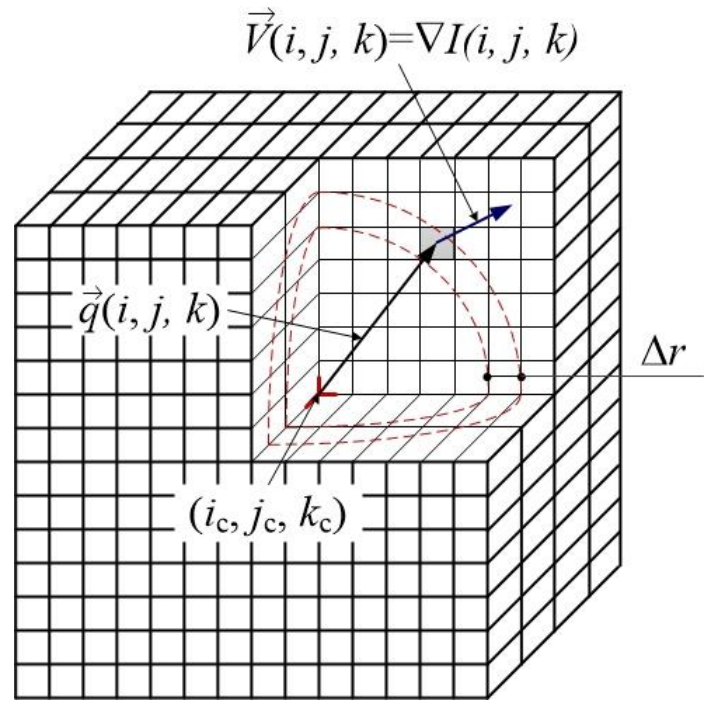

328 Figure 9: Definition of the signature function. 
330 Figure 10 illustrates an example of the signature function. The radius of the sphere

331 corresponds to the distance to the center presenting the highest value of $f(\mathrm{r})$. In the example

332 shown, the radius equals seven times the voxel size (i.e. 45.5 microns). Note that, in the 333 present work, only rounded values of radius are considered.

334

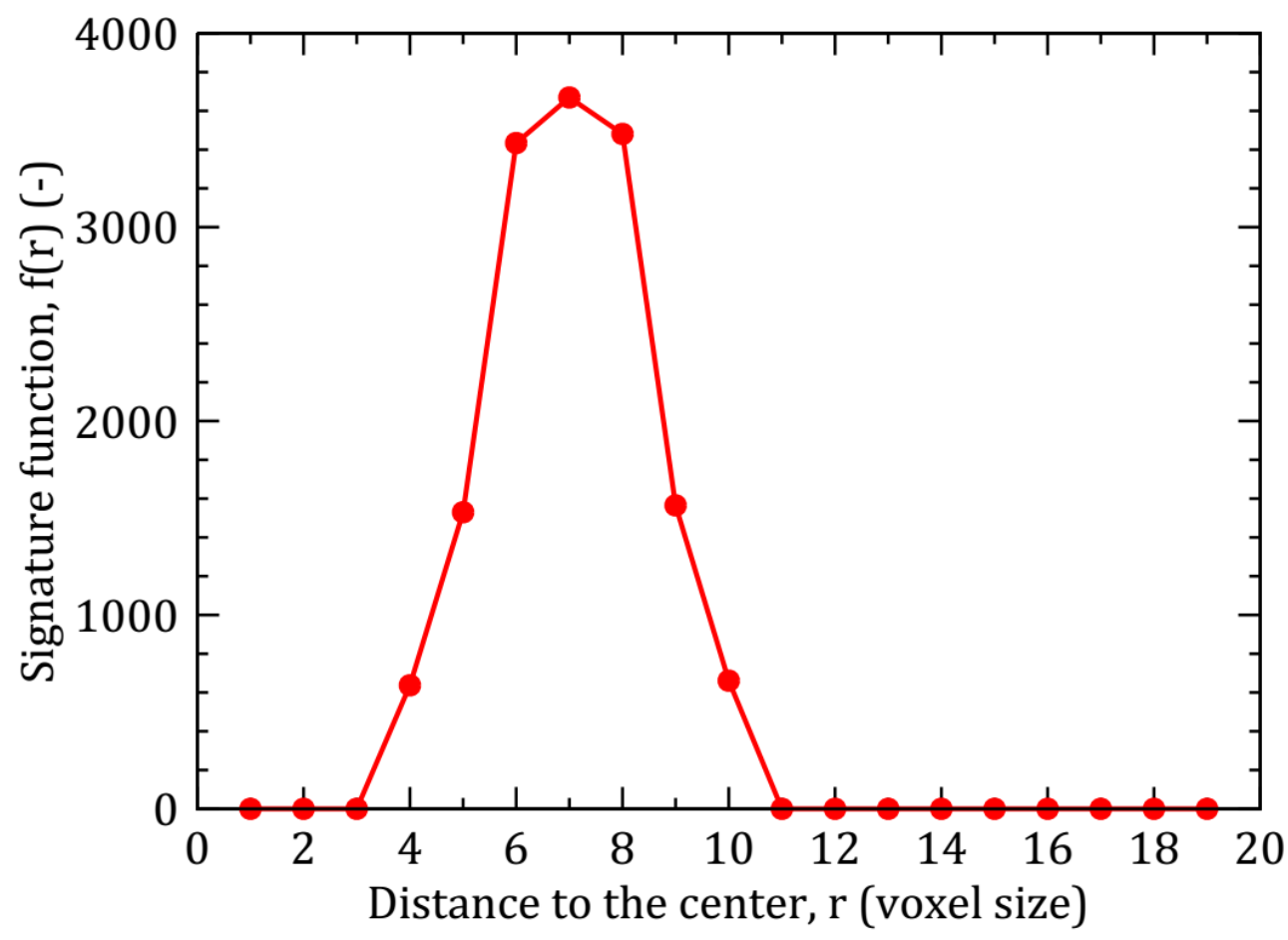

336 Figure 10: An example of signature function computed from the 3D image.

338 Figure 11 shows an example for one slice in a 3D image. The blue disks are the detected 339 spheres, and the red points represent their centers. Incomplete white disks at the border are not 340 used for the detection of the centers. 

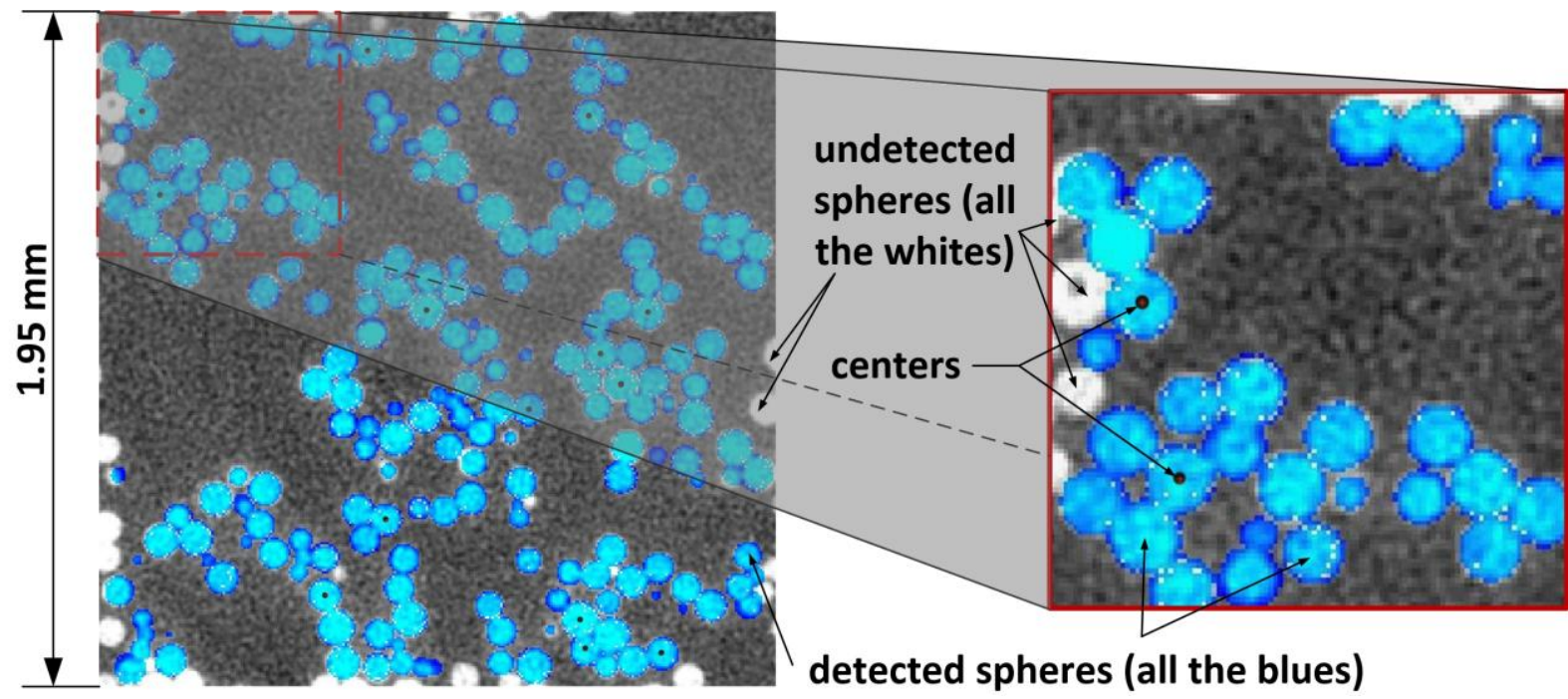

344 Figure 11: A slice in a 3D detected image.

\subsubsection{Estimating the lost contacts after the detection process}

348 As mentioned above, after the detection process, the spheres that intersect the border of the

349 cube cannot be identified (Figure 11). Hence, the contacts between these spheres and their

350 neighbors inside the SV cannot be considered. In order to estimate those lost contacts, a

351 method is proposed as follows. A new cube which is larger than the SV is created. It is called

352 the Extended Volume (or EV) with the edge length $b$ (see Figure 12), $b=a+2 d_{\max }$, where $a$

353 is the edge length of the SV, $d_{\max }$ is the maximal diameter of the detected spheres. The EVs

354 are then analyzed with the same input parameters and compared with the SVs. All grains

355 inside the SVs and the EVs having the same coordinates are first identified. The grains, inside

356 the EVs (but not identified with the SVs) can be then distinguished. 


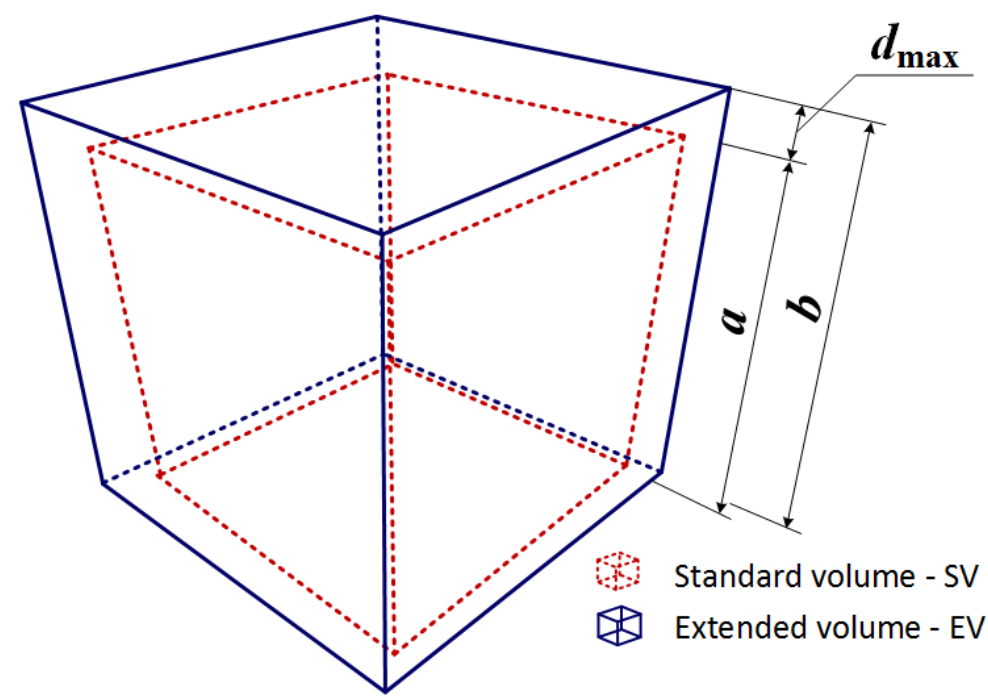

358 Figure 12: Method to find the lost contacts; the inner cube is the standard volume (SV) and 359 the outer one is the extended volume (EV).

\subsection{D images reconstruction}

Figure 13 illustrates an example of 3D images reconstruction for the SV before and after the positions of particles after the detection process. The small cubes (f) is extracted from (e). As the detected radius can only equal to rounded values of voxel size, a tolerance of 1 voxel size (6.5 microns that is $7.14 \%$ of the average grains' diameter) is then added to the grains' diameter in order to better consider the error related to the detection process. With this correction, the images (e) and (f) become (g) and (h), respectively.

Figure $13 a$ shows several large pores (up to $1 \mathrm{~mm}$ ) and stacks of spheres having dimension ranging from 0.3 to $0.5 \mathrm{~mm}$. As the average diameter of sphere is 100 micrometers, around 15 layers of voxels compose each sphere (as seen clearly in Figure 13b). 

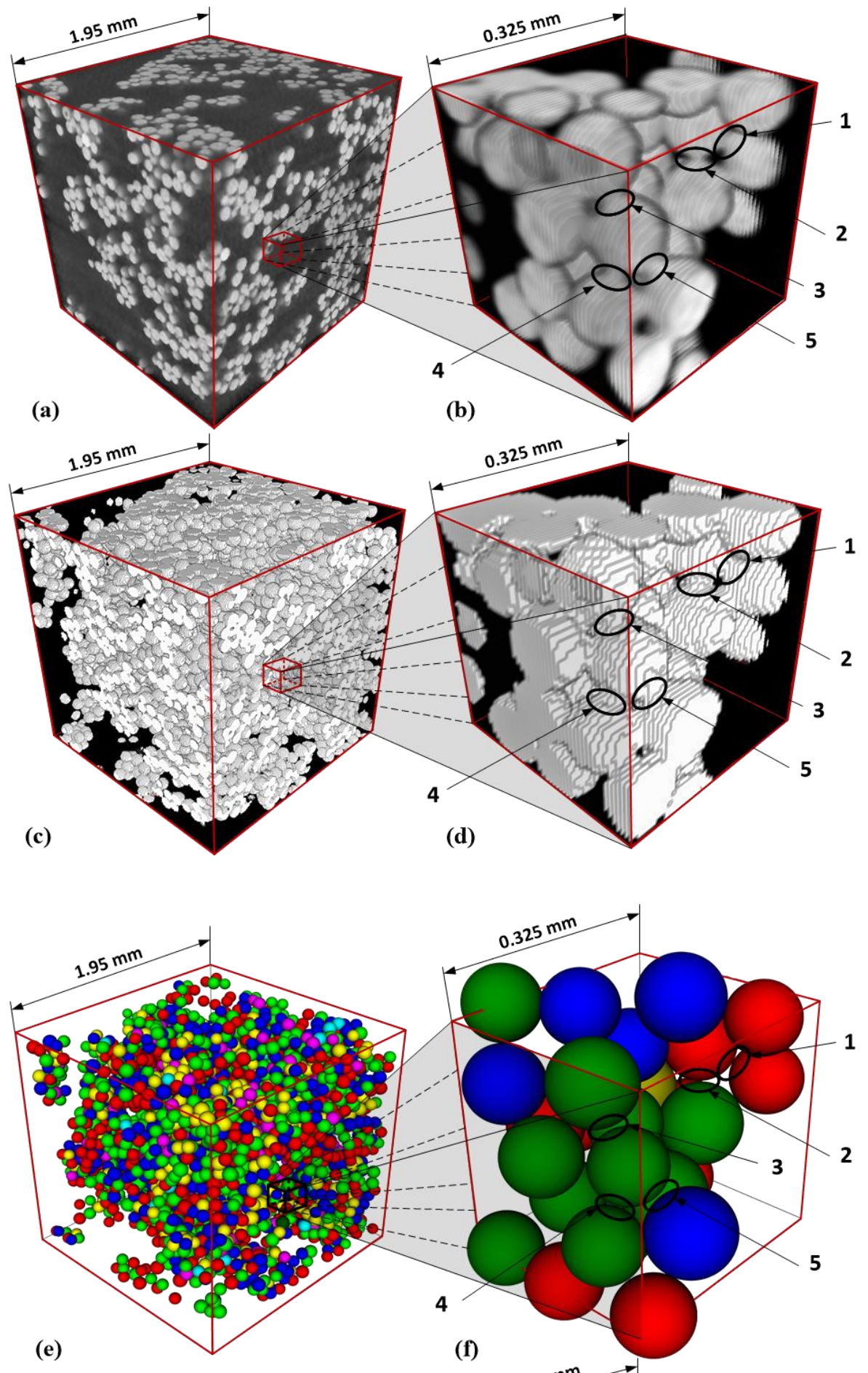

(g)

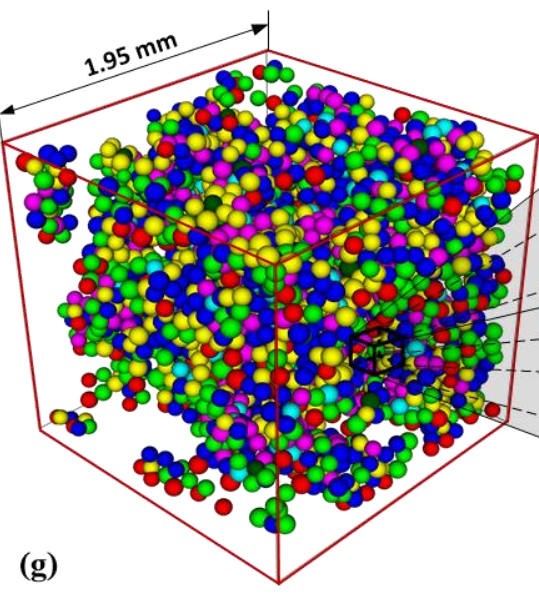

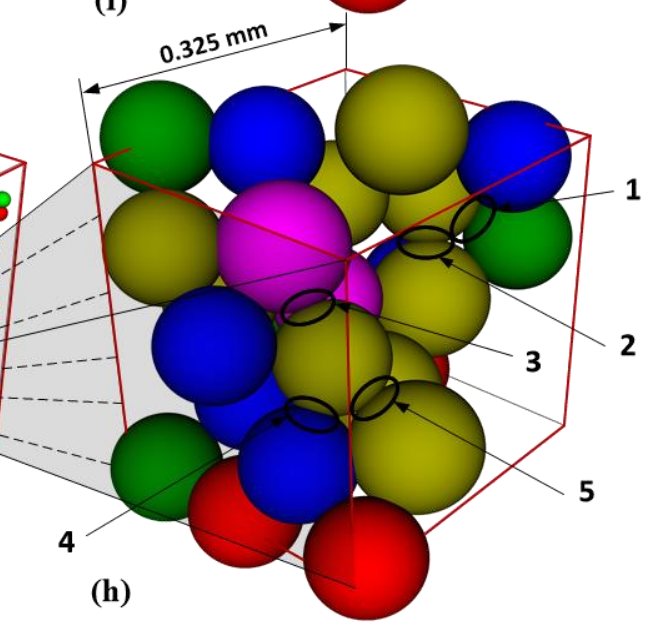


Figure 13: Example of reconstructed 3D specimens: (a, b) Original image - (c, d) Binary

380 images of the investigated cube; (e, f) Reconstructed 3D image with tolerance of 0 voxel; (g,

381 h) Reconstructed 3D image with tolerance of 1 voxel.

382

383 The contact between spheres is obvious in Figure 13(b and $d)$. Some typical contacts are 384 denoted by the positions 1 to 5 . The corresponding spheres are kept in contact at these 385 positions in the specimen after binarization (see Figure 13( $b$ and $d)$ ). However, these spheres 386 are no longer in contact after the detection process, as shown in Figure $13 f$. After adding a 387 tolerance of 1 voxel size to the diameter of all spheres, these contacts appear to be similar to 388 the ones observed before the detection. Clear evidence is shown in Figure 13h. In Figure 13(e$389 h$, the color of spheres encodes their number of contacts as follows: the red, green, blue, 390 yellow, magenta represent the spheres having zero, one, two, three, and four contacts, 391 respectively. Comparison of Figure $13 f$ and Figure $13 h$ clearly shows the effect of the change of tolerance on the detected contacts.

394 The macroscopic solid fraction and the average solid fraction of ten SVs are illustrated in

395 Figure 14. The "macro" values (red columns) are computed from the void ratio of the entire 396 specimen (Figure 2). The green columns (BD) show the average values, which were directly calculated from the binary images (before detection). The results show that the macroscopic solid fraction is different from that estimated at the center of the specimen. That can be partly explained by the heterogeneity of the microstructure of the sample (as shown in Figure 4 and

400 Figure 6). In addition, after the detection process, the solid fraction is computed by dividing 401 the volume of all particles by the volume of the cube (blue columns). The solid fraction 402 obtained after the detection process with zero tolerance $(\mathrm{AD})$ is smaller than that before the detection. However, with one voxel size tolerance (AD-Tol1), the results (magenta columns) 404 are very close to the ones before detection (green columns). This suggests that the use of a 
tolerance of one voxel size for all analyses of the 3D images after the detection process is 406 quite suitable. For this reason, in the following analyses, only the detection results with 407 tolerance of one voxel size are considered.

408

409

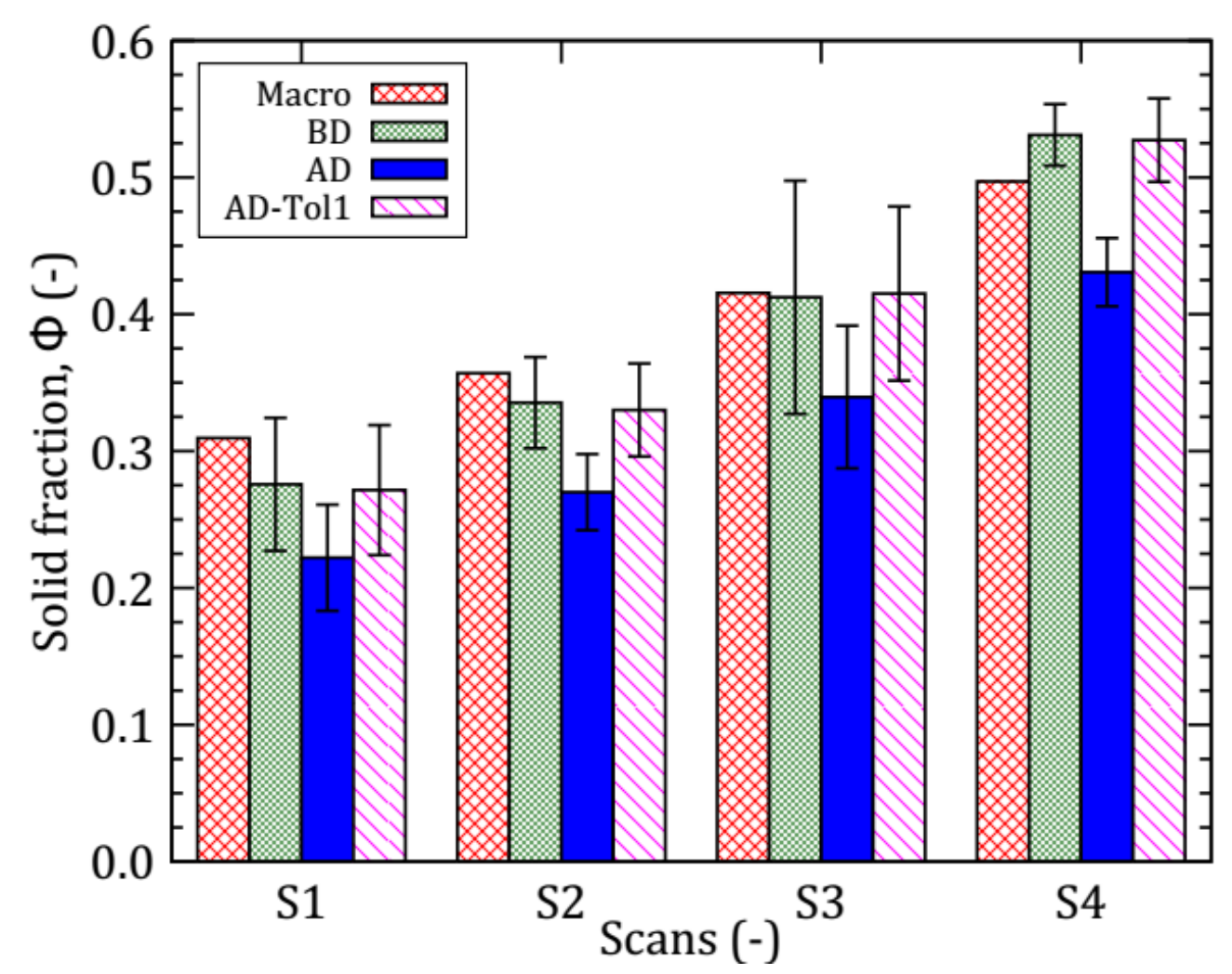

410 Figure 14: Solid fraction at different compaction levels. AD denotes after detection, BD

411 denotes before detection.

412

\section{Pore-scale characterization}

414

415 In this section, the pore-scale deformation of specimen under growing of compaction level is

416 characterized via the pore-distance distribution. To obtain this distribution, a large quantity of

417 points (about fifty times the number of particles) is randomly placed into the pore space of the

418 specimen. For each point, the shortest distance from this point to a grain is then identified. In

419 other words, this distance is the radius of a "virtual" sphere which has this point as its center

420 and has one contact point with a "real" grain. The cumulative probability of the identified 
421 distances is then computed. A statistic of the identified distances is finally collected in the

422 form of a cumulate probability. The pore distance distribution of the specimen at four

423 compaction levels is illustrated in Figure 15 in which the distance is normalized by the 424 average diameter $\langle d\rangle$ as the following equation:

$425\langle d\rangle=\frac{2 d_{\max } d_{\min }}{d_{\max }+d_{\min }}$.

426 Here, $d_{\max }$ and $d_{\min }$ are the maximal and minimal diameters of grains, respectively.

In Figure 15, at each compaction level, the gold region denotes the range of pore distance distribution (for briefness, hereafter in the section referred to as the gold region) of ten

430 different positions (ten cubes SV) in the specimen, and the continuous line denotes the 431 average value of these ten distributions. In general, the pore distances strongly decrease, $R_{\max }$ $\approx 4\langle d\rangle$ in $\mathrm{S} 1$ reduces to $R_{\max } \approx\langle d\rangle$ in $\mathrm{S} 4$, under growing of compaction level. In the loosest state S1 (Figure 15a), the gold region is rather large. That means the pore structure of the ten

$434 \mathrm{SVs}$ is different from each other. For instance, the pore having size $R \leq\langle d\rangle$ occupies approximately $40 \%$ at the lowest boundary while its percentage is about $99 \%$ at the highest boundary and is about $80 \%$ for the average value; the very large pores, $R \geq 3\langle d\rangle$, still occupy a remarkable quantity $(7 \%)$ at the lowest boundary. An example of several large pores can be clearly seen in Figure 13a. In the denser states S2 and S3 (Figure 15(b and $c)$ ), the pore distance distributions significantly decrease, $R_{\max }$ reduces to approximately $2\langle d\rangle$. In the

440 densest state S4 (Figure 15d), the gold region is rather small. This means that the pore 441 distance distribution of the ten SVs is rather similar in this state. The pore distances decrease 442 twofold from the state $\mathrm{S} 3, R_{\max } \approx\langle d\rangle$. In addition, $99 \%$ of the pores have a radius smaller 443 than $0.5\langle d\rangle$ and $50 \%$ smaller than $0.1\langle d\rangle$ in average. It should be noted that the pore 444 distribution shown in Figure 15 corresponds to that of the ten SVs, the large voids observed in 445 larger scale (Figure 4) are not considered. To assess the pore distribution of the whole specimen (shown in Figure 4), the binary x-ray 3D images can be used. 


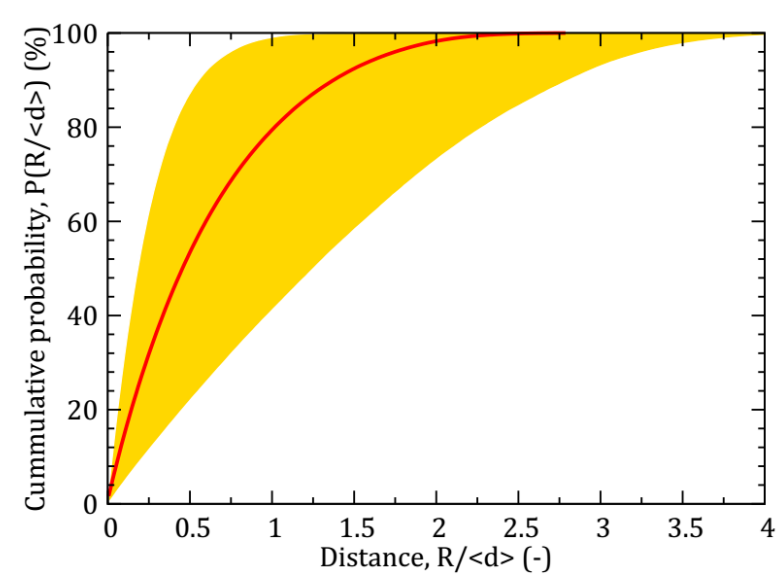

(a) $\mathrm{S} 1$

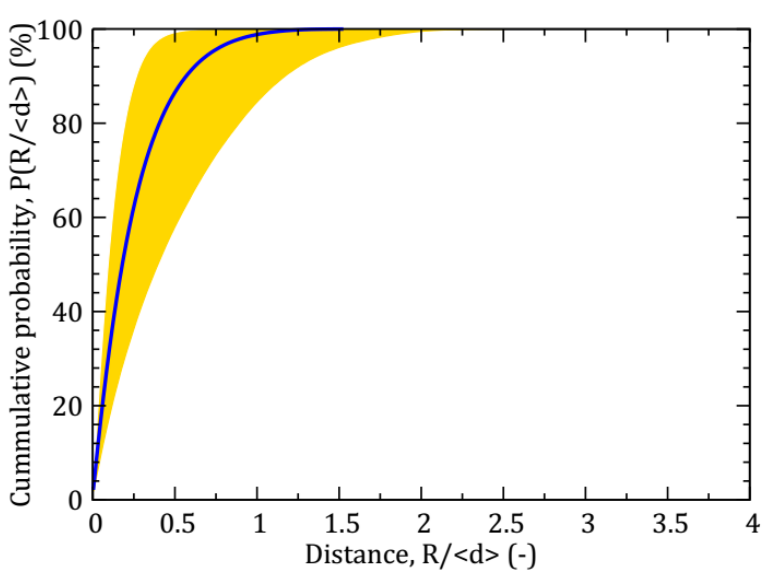

(c) S3

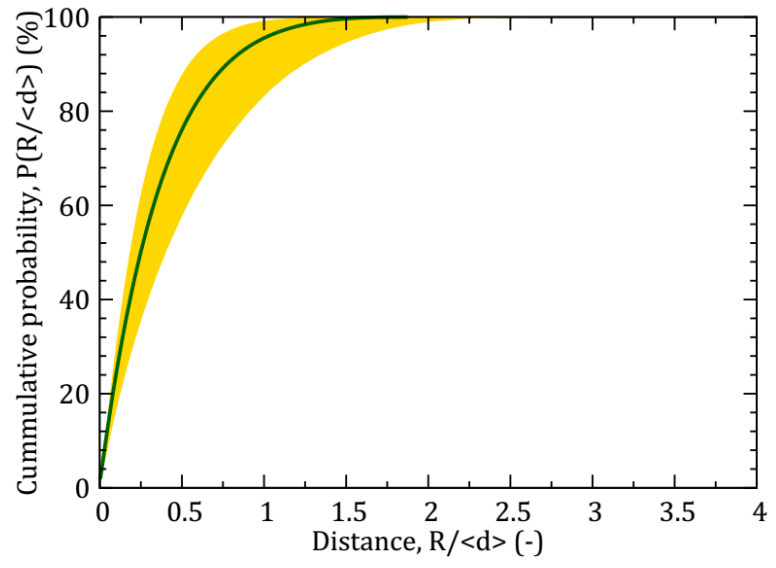

(b) $\mathrm{S} 2$

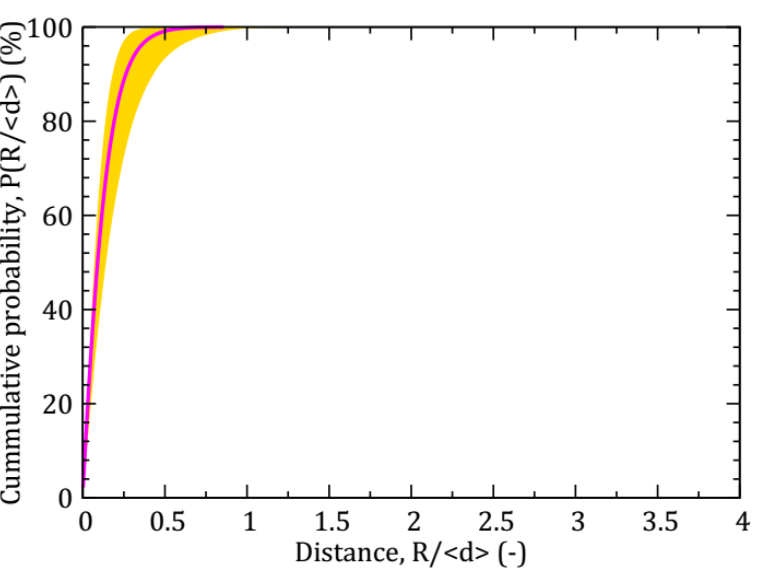

Figure 15: Pore distance distribution for four compaction levels. In each level, gold region denotes the values of ten cubes; continuous line denotes the average value.

\section{$5 \quad$ Grain-scale analysis}

The 3D reconstructed images are used in this section to perform grain-scale analysis in the SVs. As mentioned above, the agreement in solid fraction (Figure 14) suggests that the use of a tolerance of one voxel size for all analyses of the 3D images after the detection process is

460 quite suitable. In this section, only the 3D reconstructed images with tolerance of one voxel 461 size are considered. 


\subsection{Total coordination number}

464

465 In granular materials, the coordination number $z$ is defined as the average number of force466 carrying contacts per grains. If $N_{\mathrm{C}}$ is the number of force-carrying contacts between $N$ grains,

467 then the coordination number is $z=2 N_{\mathrm{C}} / N$. In the present analysis, $N_{\mathrm{C}}$ is redefined as the 468 number of pairs of contacts between $N$ grains. Pairs of contacts mean two particles that have a 469 distance between the two centers smaller than or equal to the sum of their radii. Therefore, in 470 the present part, $z$ is called the total coordination number.

471

472 Figure 16 plots the average total coordination number $z$ for the four compaction levels, S1 to 473 S4. The error bar denotes the standard deviation of ten values of ten SVs at each scan. In the 474 loosest state $\mathrm{S} 1, z$ equals to 5.29 ; it increases at higher density and reaches to 5.83 at the 475 densest state S4. $z$ remarkably varies at higher density (see the error bars in Figure 16). This 476 means that $z$ is significantly different in the ten SVs. In other words, the average value of $z$ 477 considerably depends on the structure of the specimen. As already shown in Figure 6, under 478 growing of compaction level, the void ratio in the central elevation decreases nearly twofold 479 (in the states S3 and S4) while it is negligibly decreased at the bottom elevation. This reflects 480 a strong rearrangement of the structure. In other words, the central zone of specimen is denser 481 than the bottom and the top ones. This explains why the $z$ varies more largely in the states S3 482 and S4. 


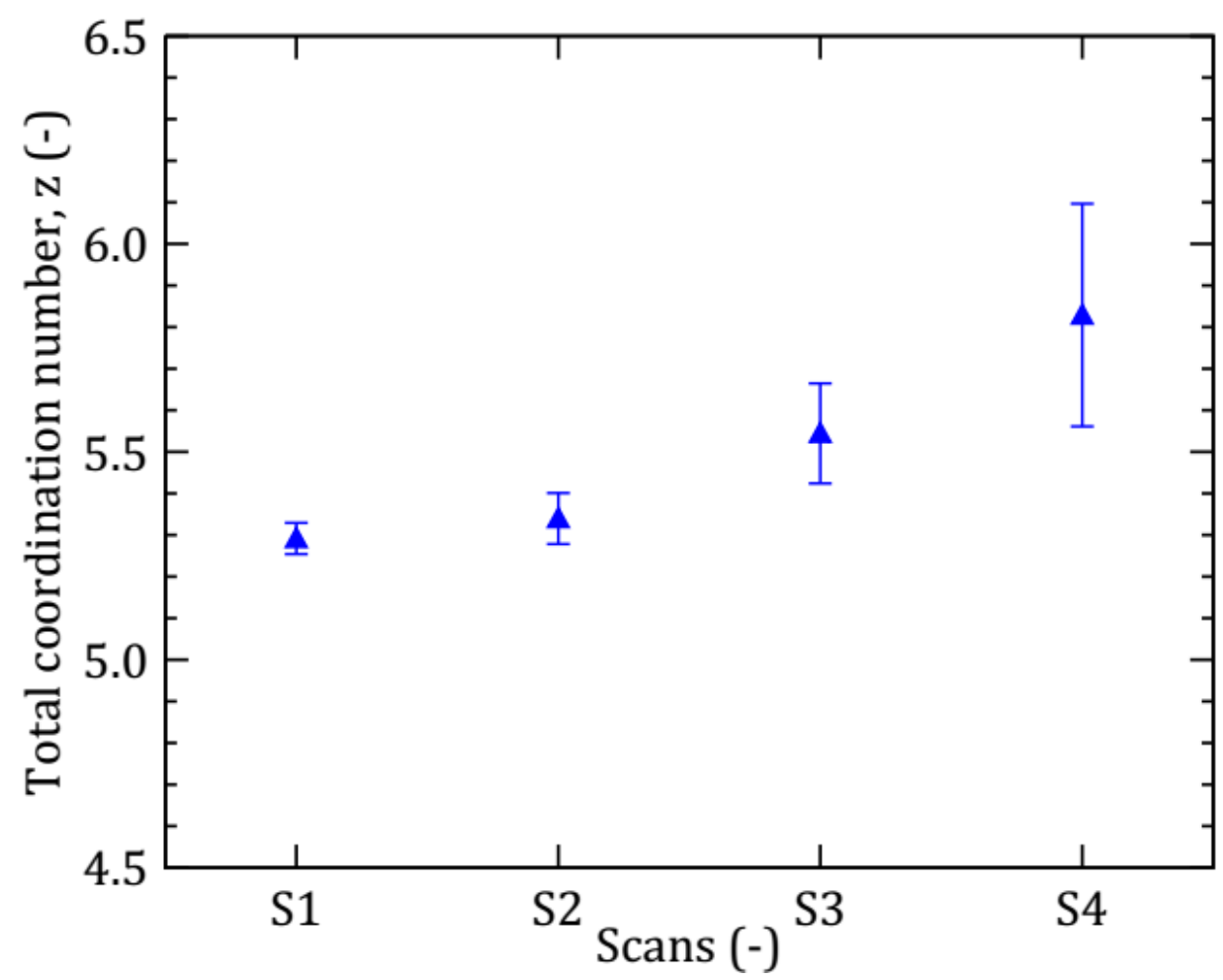

485 Figure 16: Average total coordination number for different compaction levels.

\subsection{Coordination number of close neighbors}

The coordination number of close neighbors, $z(h)$, is the average number of neighbors of one particle separated by an interstice narrower than $h$. The interparticle distance $h$ can be normalized by the average diameter $\langle d\rangle$. Figure 17 shows, for the four compaction levels, $z(h)$ versus the interparticle distance between grains; this distance varies between 0 and 1 voxel

493 size (that is $0 \leq h /\langle d\rangle \leq 0.07$ ). The color region represents the variation of ten SVs, the continuous line represents the average value. At $h=0, \mathrm{z}(h=0)$ is equal to $z$ (see Figure 16). At the loosest state $\mathrm{S} 1, z(h)$ increases from 5.29 to 5.75 (about $9 \%$ ) while it increases from 5.83 to 7.16 (approximately $23 \%$ ) at the densest state S4. Actually, this increased rate reflects the structure of the specimen. This can be explained via the decrease in the pore distance distribution, as depicted in Figure 15. Under growing of compaction level, when the specimen is compressed, the large pores are strongly decreased (e.g. $R_{\max } /\langle d\rangle=4$ downs to $R_{\max } /\langle d\rangle=1$,

500 from the $\mathrm{S} 1$ to the $\mathrm{S} 4)$. This means that the specimen becomes denser. That explains why the 
501 number of close neighbors at the denser states increases more quickly than the one at the

502 looser states. In addition, similar to $z$, the variation of $z(h)$ at the denser states is larger than

503 the one at looser states.

504

505

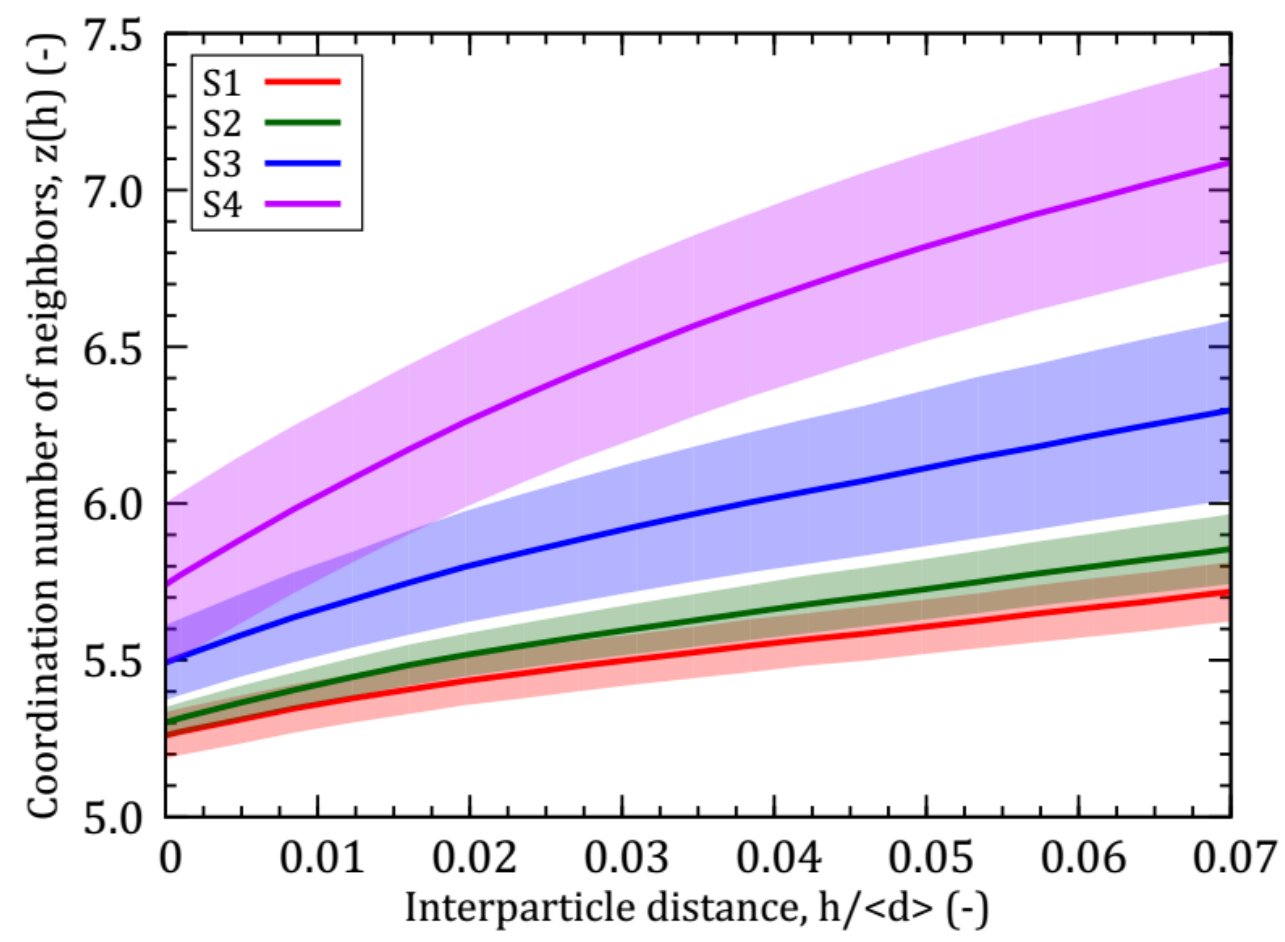

506 Figure 17: Average coordination numbers of close neighbors for different compaction levels.

507

\section{$508 \quad 5.3 \quad$ Number of contacts per grain}

510 Figure 18 shows the average number of contacts per grain for the four compaction levels. In

511 general, the grains have zero contact to eleven contacts, and the fraction of grains having two

512 or three contacts are predominant, i.e. 20 - $24 \%$ for each of these categories. For the denser

513 state, the occurrence of high values of $N_{\mathrm{C}}\left(N_{\mathrm{C}} \geq 3\right)$ is higher than in the other states while at

514 low value of $N_{\mathrm{C}}\left(N_{\mathrm{C}}<3\right)$, this occurrence is lower than in the looser states. This confirms the

515 influence of the granular structure on the contacts of grains. Under growing of compaction

516 level, the specimen's structure becomes denser and more contacts are created. Theoretically,

517 the maximal number of contacts per grain is 12 . In the present case, $N_{\mathrm{C}}=11$ is the maximal 
518 value and it occupies a minimal probability, nearly $0.003 \%$ (S3) and $0.007 \%$ (S4). This

519 probability only occurs in the denser states (S3 and S4) and it is equivalently a few grains

520 having 11 contacts. $N_{\mathrm{C}}=0$ still occupies a significant percentage, approximately $9.0 \%$ to 9.5

$521 \%$. In reality, the grain having zero contact does not exist in the granular structure. However,

522 in the present case, a remarkable quantity of the grains having zero contact appears after the 523 detection process.

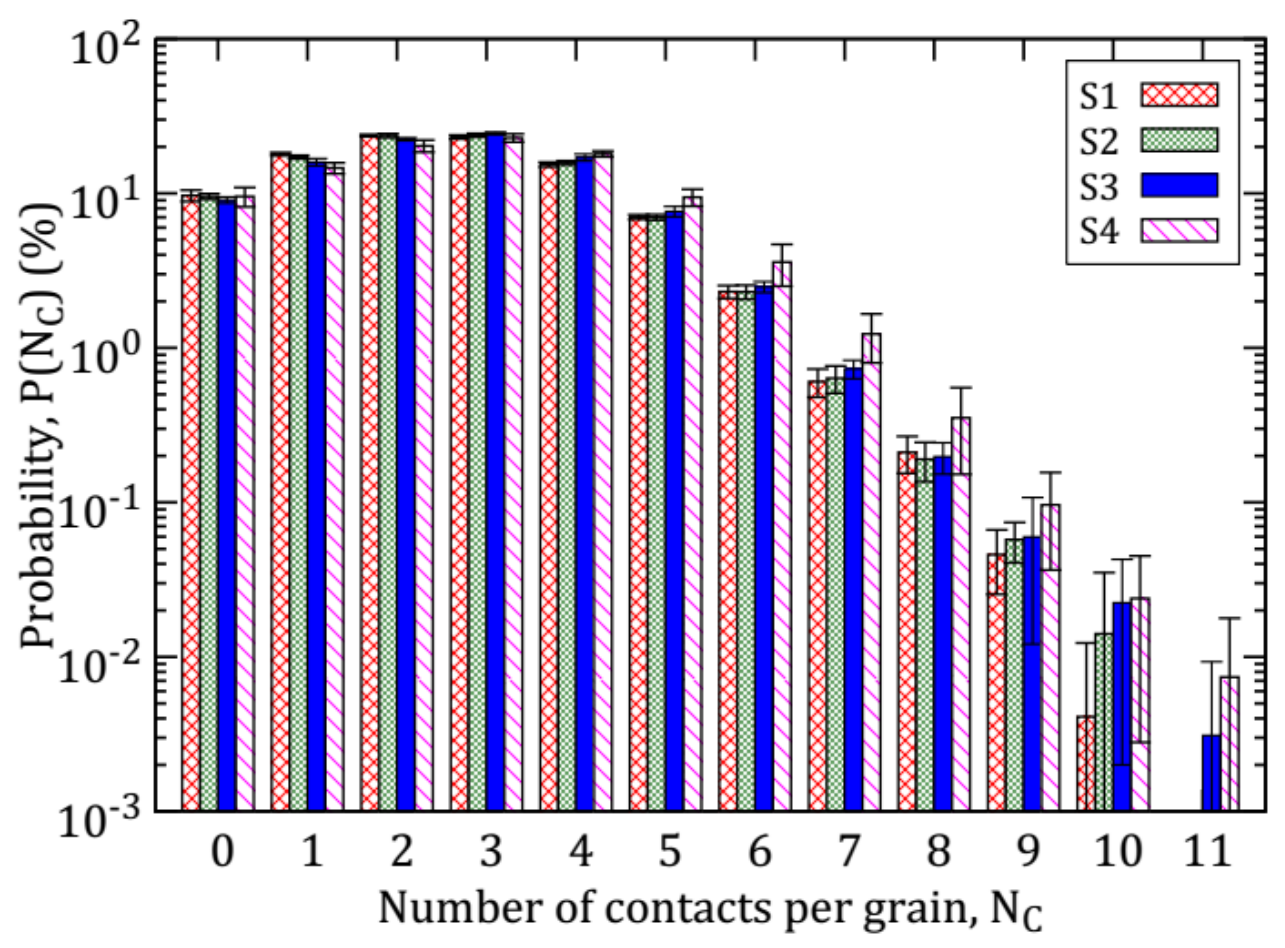

526 Figure 18: Average number of contacts per grain for different compaction levels.

528 Interestingly, spheres with one contact can be found in this structure, one example is

529 illustrated in Figure 19. This is a small cube extracted from the Figure 13a. For these cases,

530 after the detection process, these spheres have either one or zero contact. The presence of

531 water menisci and the possible rolling resistance [52] at contact between grains could explain

532 this. Indeed, with an average diameter of 100 microns, the particle weight loses relevance

533 with respect to capillary forces [53], which could alone stabilize these grains. Besides, as the 
534 surface of the grain is not really perfect (see Figure 1), the rolling resistance can be 535 significant.

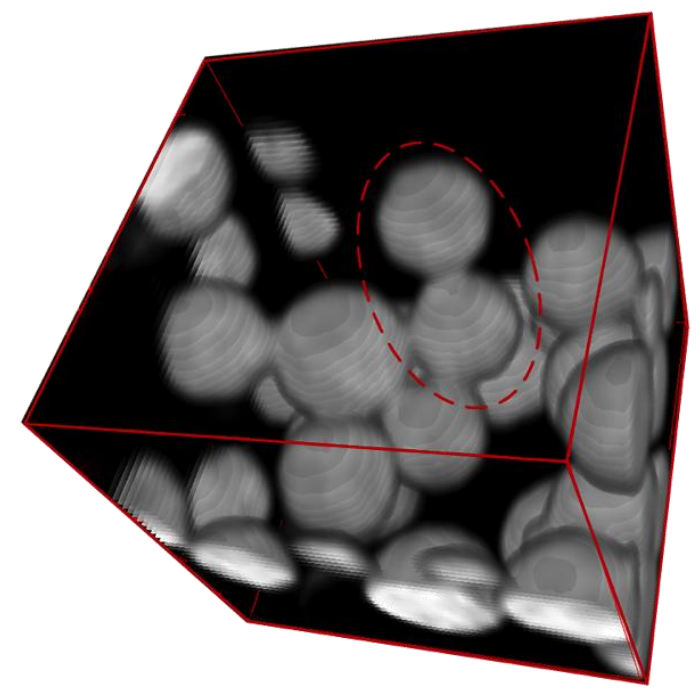

538 Figure 19: An example of a sphere having one contact.

\subsection{Radial distribution function}

541

542 The radial distribution function, $g(r)$, is the probability of finding a particle center in a

543 spherical shell of a radius $r$, given that there is another particle at the center of the spherical 544 shell [54]. $g(r)$ is related to the average number of particles by

$545 p\left(r_{2}\right)-p\left(r_{1}\right)=\int_{r_{1}}^{r_{2}} g(r) 4 \pi r^{2} d r$, (Erreur ! Signet non défini.)

546 where $p(r)$ is the average number density of particles in a spherical shell of radius $r$. In this 547 formula, radial distances are normalized by the average diameter $\langle d\rangle$ (given by Eq. (3)).

549 Figure 20 shows $g(r)$ for the four compaction levels. The radial distance is normalized by the average diameter $(r /\langle d\rangle)$. The curves for the various states are nearly similar in their

551 oscillations and peaks. $g(r)$ starts from a maximum value at $r=\langle d\rangle$ with different amplitudes;

552 the loosest system presents the highest amplitude. $g(r)$ then decreases to minimum probability 

at $r=1.2\langle d\rangle, r=1.3\langle d\rangle, r=1.35\langle d\rangle$, and $r=1.4\langle d\rangle$ for S1, S2, S3, and S4, respectively.

554 It increases subsequently to a local peak at $r \approx 1.9\langle d\rangle$. From this peak, $g(r)$ continues to 555 fluctuate slightly until it approaches a rather constant value at $r=3.0\langle d\rangle$.

556

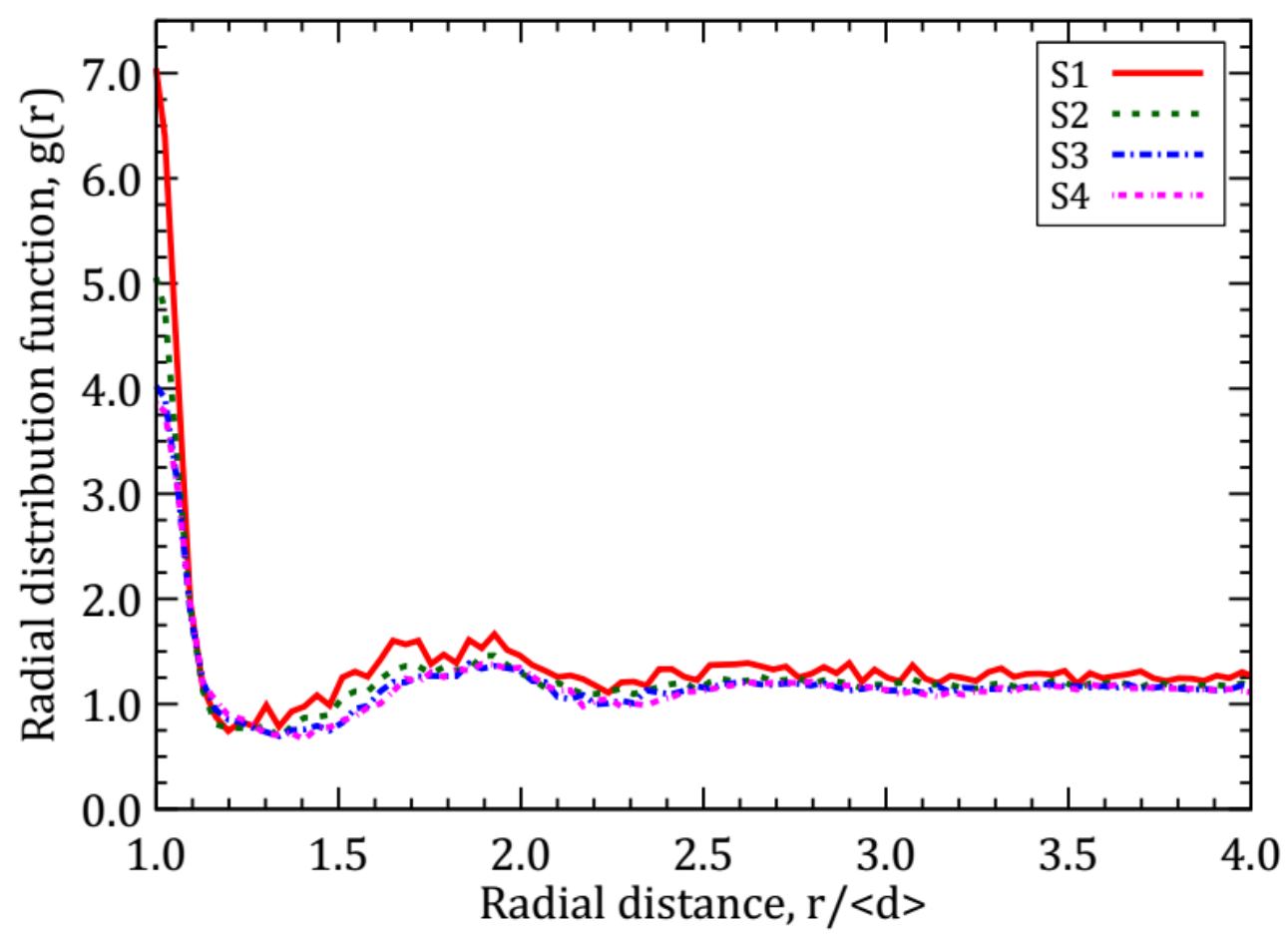

557

Figure 20: Radial distribution functions for particle centers of four compaction levels.

$560 g(r)$ is a useful tool to describe the structure of a system, and it has been used widespread in

561 the study of granular materials, especially in the numerical simulations $[55,56]$. At very

562 short $r(r /\langle d\rangle<1) g(r)$ must equal zero, because two particles cannot occupy the same space.

563 It is clear that $g(r)$ should go to 1 for large $r . g(r)$ shown in Figure 20 goes indeed 564 asymptotically to 1 for large $r$. As mentioned above, these analyses are performed on the ten 565 SVs (virtual cubical specimens with edge length $=1.95 \mathrm{~mm}$ ). At this scale, the results would 566 suggest a representative volume element (RVE) size of about 3d (300 micron). However, 567 characterization of the sample's homogeneity, based on the asymptotic behavior method for 568 air and solid fractions [57], would give a better knowledge on the heterogeneity of the whole specimen (10 $\mathrm{mm}$ in height and $20 \mathrm{~mm}$ in diameter). 


\section{$571 \quad 5.5 \quad$ Contact network}

572

573 Figure 21 illustrates the contact networks (on the left-hand side) and the reconstructed 3D

574 image (on the right-hand side) of a typical SV (for each compaction level) represented in a 575 narrow slice having a thickness of three times the grain diameter $(3\langle d\rangle \approx 40$ voxel sizes). The 576 contacts between particles are represented by segments of different colors joining particle 577 centers in two types (on the left-hand side of Figure 21): (i) the red lines link the center of two 578 spheres in contact; (ii) the blue lines link the center of two spheres having an interparticle 579 distance higher than zero but smaller than 1 voxel size (6.5 microns).

580

581 Under the lowest compaction level S1, structure exhibits strands (or chains) of single particles 582 - see the rectangle regions of Figure 21( $a$ and $b)$. These chains still appear in the higher 583 compaction level S2 (Figure 21(c and $d)$ ). This could be explained by the appearance of 584 rolling resistance at contacts between grains as mentioned above. This resistance tends to 585 react the collapse of structure under external applied forces. However, under higher 586 compaction levels, these contacts are broken and the specimen is restructured; The chains of 587 single particles no longer exist (see Figure 21(e-h)). This phenomenon is also observed in a 588 numerical study of model cohesive grains using discrete element method [58]. 


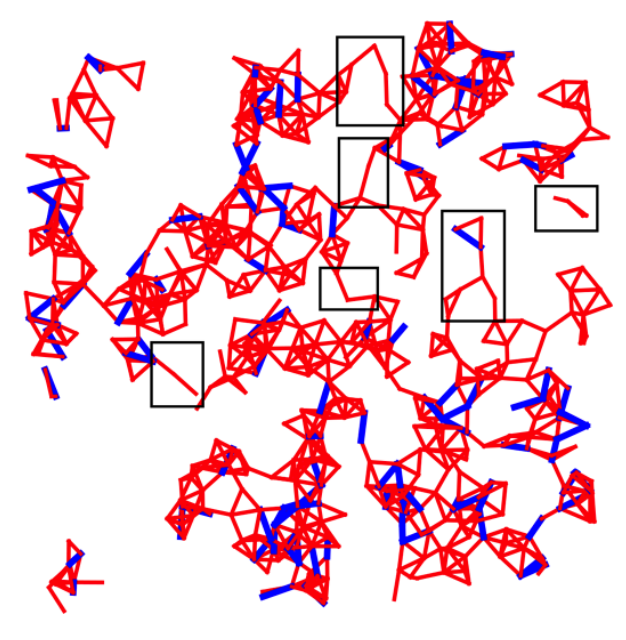

(a) $\mathrm{S} 1$

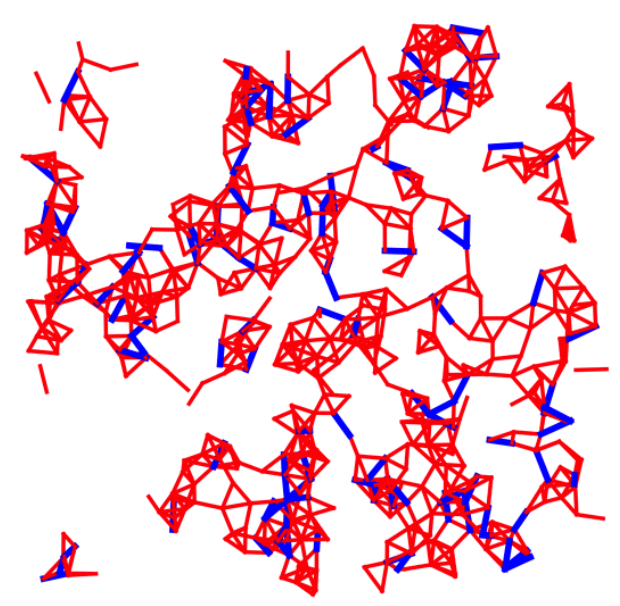

(c) $\mathrm{S} 2$

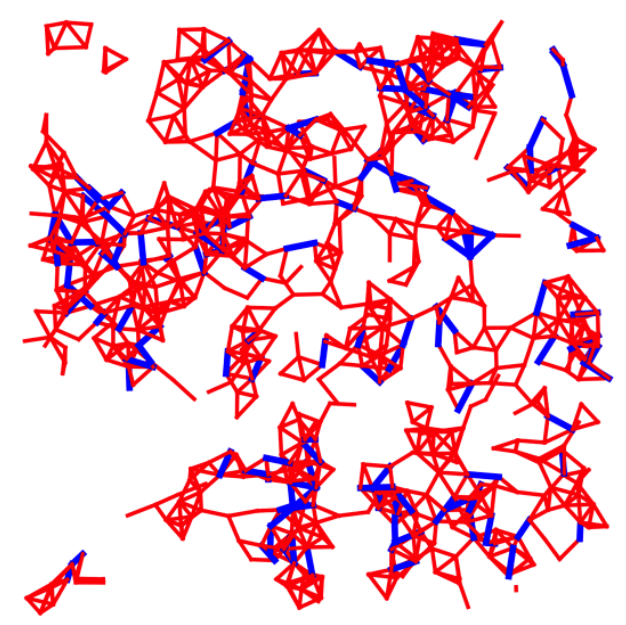

(e) $\mathrm{S} 3$

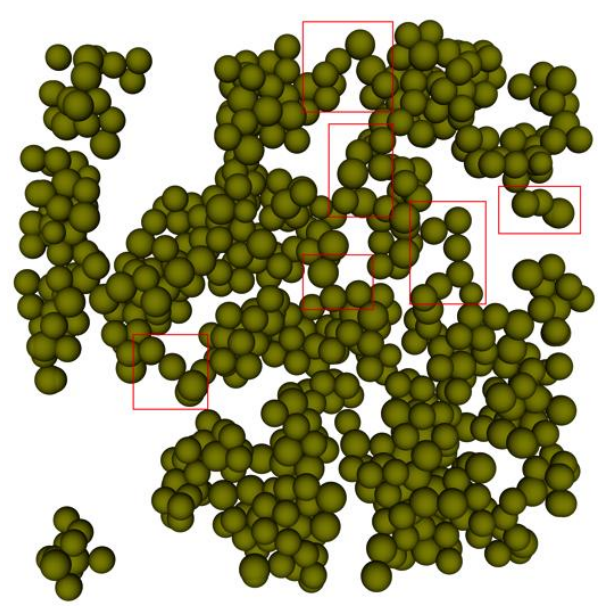

(b) $\mathrm{S} 1$

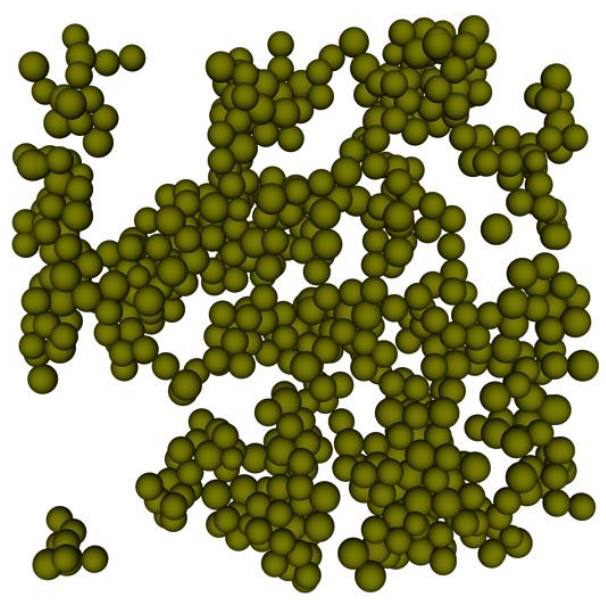

(d) S2

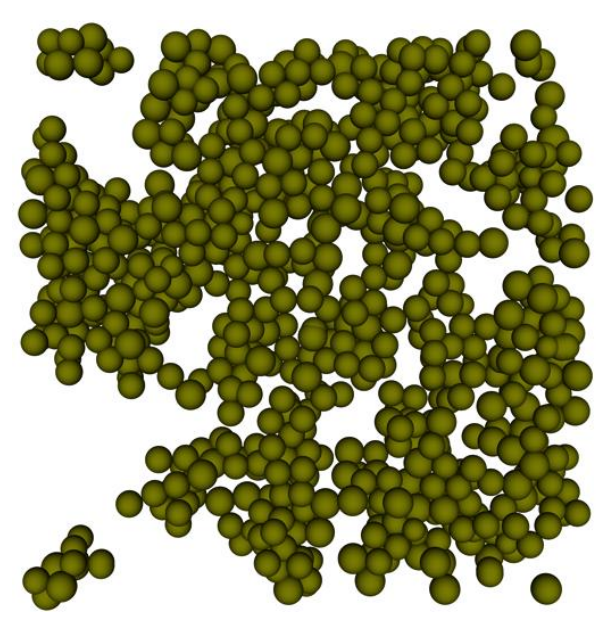

(f) $\mathrm{S} 3$ 


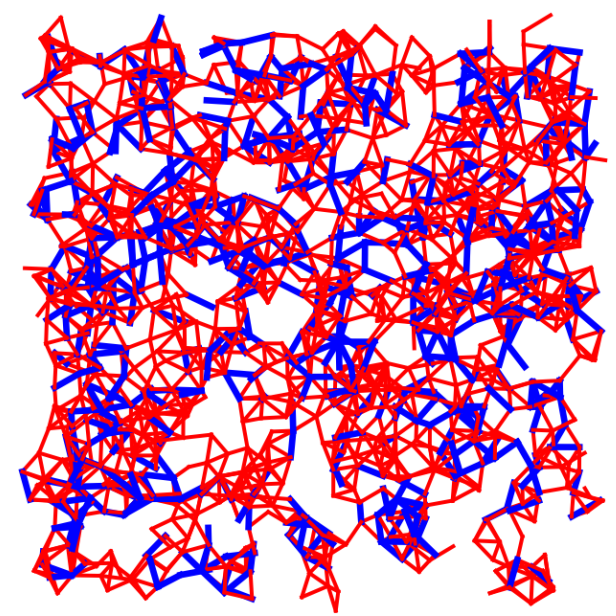

(g) S4

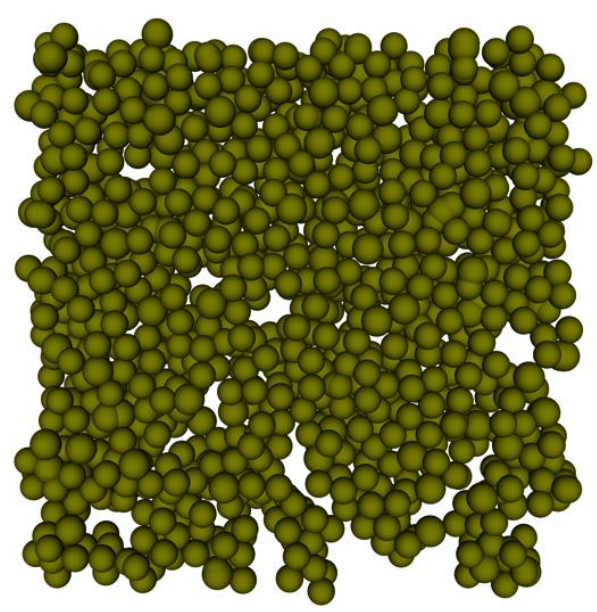

(h) S4

591 Figure 21: Contact network and reconstructed 3D image of a typical slice under growing of 592 compaction level.

593

\section{Discussion}

595

596 A very loose yet stable-state of wet granular material was produced in laboratory even using 597 very small water content. Evidently, the structure of the specimen is heterogeneous but it 598 seems very difficult to make the specimen more homogeneous using such a low density.

600 The compression curves obtained show a 3-stages shape (see Figure 2). Following the usual 601 concept of soil mechanics [5, 59], there are only two stages 1 and 2 on the oedometric 602 compression curve. Interestingly, the stage 3 is also described in the S-shape curves obtained 603 on agricultural soils [59]. In this stage, the void ratio is slightly decreased and maintained 604 nearly constant at high stress (see Figure 2). That confirms the remarkable effect of the elastic 605 deformation of grains at the contact points.

606 
607 In the work of Than et al. [12], numerical simulations (by the discrete element method) of a

608 model granular assembly, made of spherical balls, were used to investigate the influence of a

609 small amount of an interstitial wetting liquid, forming capillary bridges between two adjacent

610 particles on the plastic response in isotropic compression. The study was restricted to the

611 pendular state of low saturation (degree of saturation smaller than $1 \%$ ), in which the wetting

612 liquid is confined in bonds or menisci joining contacting grains. By using glass beads of 115

$613 \mu \mathrm{m}$ (similar to those in the present work), a loose granular structure (void ratio equals 2.30)

614 was created. Isotropic compression behavior of this structure showed equally a 3-stages

615 shape. The agreement between the results obtained by the two methods (experiments in the

616 present works and numerical simulation in the work of Than et al. [12]) confirm the key role

617 of water menisci that stabilize the structure of specimen at very loose state. This statement is

618 also in agreement with the results of Scheel et al. [41] showing a sharp rise of tensile strength

619 of assemblies of wet glass spheres at very small water content.

620

621 In the present work the position and radius of the spheres were detected by the algorithm

622 initially developed by Xie et al. [47] for 3D images, modified following Peng et al. [48]

623 (proposed for 2D images) along with the Adaptive Hough Transform algorithm of Illingworth

$624 \&$ Kittler [49]. Within this method, integer number of pixels was used to set the radius of 625 particles, which would induce error on the characterization of the interparticle contacts. The 626 results would be significantly improved by using the kalisphera-based technique [33] for 627 sphere-matching in real 3D images of spheres. Besides, detecting interparticle contacts from 628 x-ray microtomography 3D images by using a greyscale threshold that selects the correct solid 629 volume would induce also error [60]. For the sake of simplicity, in the present work, the 630 contacts were detected from the re-constructed 3D images and the above limitations should be 631 considered when assessing the results. 
633 The main contribution of the present work would be the links between macro and microscopic

634 behaviors of specimen under growing of compaction level (Figure 2, Figure 6, Figure 14-

635 Figure 17). In the stage 1 of the compaction curve (Figure 2), the void ratio remains nearly 636 constant and slightly decreases at the end of this regime (equivalently the position of the scan

637 S1). Under growing of stress in stage 2, the specimen structure quickly collapses. This is 638 evidently reflected by a twofold increase in solid fraction from the loosest state (S1) to the 639 densest state (S4) (see Figure 14) or by a twofold decrease of void ratio in the central layers of 640 the specimen (see Figure 6). Besides, the strong decrease in the pore distance distributions at 641 four compaction levels (see Figure 15) appropriately demonstrates this collapse. In the state 642 S3, the structure is significantly disturbed. This is obviously confirmed via the largest error 643 bar of solid fractions at the S3 (see Figure 14), the large gold region (see Figure 15c) and the 644 void ratio distribution (blue curve in Figure 6). At higher densities, the number of contacts is 645 larger than the one at lower densities (see Figure 16). In the stage 3 of the compaction curve, 646 starting from S4, the structure collapses negligibly because of the elastic deformation of 647 grains at contact regions. This is demonstrated by the highest density of S4 and the slight 648 increase of $z$ (see Figure 16 and Figure 17).

650 Moreover, the pore-scale deformation of specimen under growing of compaction level is 651 clearly observed in Figure 15 and Figure 21. Initially, the structure can be stabilized in very 652 loose state with a significant quantity of very large pores (see Figure 15a and Figure 21( $a$ and $653 b)$ ). Several marked rectangle regions in Figure 21( $a$ and $b$ ) show chains of single particles. 654 Such a structure cannot exist and stabilize without rolling resistance at contacts [58]. Under 655 growing of compaction level the structure quickly collapses and the pores size remarkably 656 decreases. In the densest state $\mathrm{S} 4$, the pores having size $\mathrm{R} \geq 0.5\langle d\rangle$ (equivalently the grain 657 size) occupy a tiny amount (see Figure $15 d$ ), and they can be seen in Figure 21( $g$ and $h$ ). 
659 Concerning the grain-scale investigations, several authors reported the distribution of number

660 of contacts (i.e. the coordination number) [27, 35, 61-63] by using different methods to detect

661 the contacts between the spheres. However, calculating the number of contacts is difficult due

662 to the uncertainty when calculating the accurate position of the center of the particle,

663 especially with the small size of grains and low resolution of the tomography images. That is

664 why the tolerance of 1 voxel size is chosen for all calculations in the present study. This 665 tolerance is very close to the interval tolerance of $(1-2)^{1 / 3}$ voxel sizes used by Aste et al. [34,

666 62] while their size of particles is bigger than that in the present work and the resolution is not 667 too much different. In several packing studies $[35,38,62]$, the spheres are in contact with 11 668 particles at most and with 2 at least. Meanwhile, in the present study, the maximum number 669 of contacts per grains is also 11 with a tiny percentage $(0.03 \%$ and $0.07 \%)$ but this maximum 670 value only occurs in the denser states. Besides, there is still a significant percentage of spheres 671 that have zero (around 9.5\%) contact which should not exist theoretically. As mentioned 672 previously, the rounded values of the detected spheres remarkably affect the accuracy of these 673 analyses.

674

6757 Conclusion

676 A very loose structure of granular materials (glass beads) is experimentally created thank to 677 the presence of a small quantity of water forming capillary bridges. One-dimensional 678 compression tests combined with X-ray computed tomography observations allow addressing 679 the following conclusions:

680

681 Firstly, the macroscopic behavior of wet beads at loose state (initial void ratio of about 2.30) 682 obtained from oedometric compression tests has usually three regimes: $(i)$ at stresses lower 683 than the apparent pre-compression stress, the initial structure sustains the applied load with a 684 very small rearrangement; (ii) when the stresses exceed the pre-compression stress, the loose 
structures collapse and restructure; and (iii) at higher stresses, the elastic deformation of

686 grains at contact points dominates the specimen deformation. The XRCT observations (global

687 scans) show that the initial loose structure is heterogenous, consisting of large voids, and its

688 behavior during the second regime is mainly related to the structure rearrangement around

689 these large voids.

690

691 Second, the position and radius of the spheres were detected by combining various algorithms

692 proposed in the literature. Within this method, integer number of pixels was used to set the 693 radius of particles. From the 3D reconstructed images, several fundamental microstructural 694 properties are obtained such as coordination number, number of close neighbors, distribution 695 of number of contacts, radial distribution function, contacts network. Changes of these 696 properties at various stages of the compression curve allow better understanding the main 697 mechanisms at grain-scale level that influence the behavior at the pore scale and the 698 macroscopic scale. Although this experimental approach still needs to be improved, in 699 comparison with some existing numerical and experimental works, it provides an appropriate 700 characterization of the grain-scale behavior of wet granular soils at very loose states during 701 compression.

702

703 Finally, our results emphasize the key role of water menisci that stabilize the structure of 704 specimen at very loose state as well as the appearance of rolling resistance at contact between 705 grains. Indeed, the intrinsic compression behavior is due to the collapse of pore's structure 706 and the rearrangement of grains in the specimen under growing of external force. 
710 This work is part of the first author's Ph.D. thesis funded by the Ministry of Education and

711 Training of Vietnam. The authors are grateful to Dr. Michel Bornert (Laboratoire Navier) for

712 his useful suggestions and Mr. Jean-Marc Plessier (Laboratoire Navier) for the scanning

713 electronic microscopic image of a glass bead.

714

\section{References}

716 1. Wood DM (1990) Soil behaviour and critical state soil mechanics. Cambridge University $717 \quad$ Press

2. Mitchell JK, Soga K (1976) Fundamentals of Soil Behavior. Wiley

3. Pierrat P, Caram HS (1997) Tensile strength of wet granular materials. Powder Technol 91:83-93. doi: 10.1016/S0032-5910(96)03179-8

4. Fournier Z, Geromichalos D, Herminghaus S, et al. (2005) Mechanical properties of wet granular materials. J Phys Condens Matter 17:477-502. doi: 10.1088/0953$8984 / 17 / 9 / 013$

5. Munõz-Castelblanco J, Delage P, Pereira J-M, Cui YJ (2011) Some aspects of the comression and collapse behaviour of an unsaturated natural loess. Géotechnique Lett 1:17-22. doi: 10.1680/geolett.11.00003

6. Jiang M, Hu H, Liu F (2012) Summary of collapsible behaviour of artificially structured loess in oedometer and triaxial wetting tests. Can Geotech J 1157:1147-1157. doi: $10.1139 / \mathrm{T} 2012-075$

7. Mitarai N, Nori F (2006) Wet granular materials. Adv Phys 00:1-50.

8. Bruchon J-F, Pereira J-M, Vandamme M, et al. (2013) Full 3D investigation and characterisation of capillary collapse of a loose unsaturated sand using X-ray CT. Granul Matter 15:783-800. doi: 10.1007/s10035-013-0452-6 
10. Delenne J-Y, El Youssoufi MS, Cherblanc F, Bénet J-C (2004) Mechanical behaviour and failure of cohesive granular materials. Int J Numer Anal Methods Geomech 28:15771594. doi: 10.1002/nag.401

11. Delenne J-Y, Soulié F, El Youssoufi MS, Radjai F (2011) From liquid to solid bonding in cohesive granular media. Mech Mater 43:529-537. doi: 10.1016/j.mechmat.2011.06.008

12. Than V-D, Khamseh S, Tang A-M, et al. (2016) Basic Mechanical Properties of Wet Granular Materials: A DEM Study. J Eng Mech C4016001. doi: 10.1061/(ASCE)EM.1943-7889.0001043

13. Sweijen T, Nikooee E, Hassanizadeh SM, Chareyre B (2016) The Effects of Swelling and Porosity Change on Capillarity: DEM Coupled with a Pore-Unit Assembly Method. Transp Porous Media 113:207-226. doi: 10.1007/s 11242-016-0689-8

14. Sweijen T, Chareyre B, Hassanizadeh SM, Karadimitriou NK (2017) Grain-scale modelling of swelling granular materials; application to super absorbent polymers. Powder Technol 318:411-422. doi: https://doi.org/10.1016/j.powtec.2017.06.015

15. Chalak C, Chareyre B, Nikooee E, Darve F (2017) Partially saturated media: from DEM simulation to thermodynamic interpretation. Eur J Environ Civ Eng 21:798-820. doi: $10.1080 / 19648189.2016 .1164087$

16. Melnikov K, Wittel FK, Herrmann HJ (2016) Micro-mechanical Failure Analysis of Wet Granular Matter. Acta Geotech 11:539-548.

17. Delenne J-Y, Richefeu V, Radjai F (2015) Liquid clustering and capillary pressure in granular media. J Fluid Mech 762:R5-1-R5-10. doi: 10.1017/jfm.2014.676

18. Gilabert F, Roux J-N, Castellanos a. (2008) Computer simulation of model cohesive powders: Plastic consolidation, structural changes, and elasticity under isotropic loads. Phys Rev E 78:031305. doi: 10.1103/PhysRevE.78.031305

19. Kadau D, Bartels G, Brendel L, Wolf DE (2003) Pore Stabilization in Cohesive Granular Systems. Phase Transitions 76:315-331. doi: 10.1080/0141159021000051460 
20. Khamseh S, Roux J-N, Chevoir F (2015) Flow of wet granular materials: a numerical study. Phys Rev E 92:022201-19. doi: 10.1103/PhysRevE.92.022201

21. Richefeu V, Radjaï F, Youssoufi MS El (2006) Stress transmission in wet granular materials. Eur Phys J E 21:359-369.

22. Richefeu V, El Youssoufi MS, Azéma E, Radjaï F (2009) Force transmission in dry and wet granular media. Powder Technol 190:258-263. doi: 10.1016/j.powtec.2008.04.069

23. Rognon PG, Roux J-N, Wolf D, et al. (2006) Rheophysics of cohesive granular materials. Europhys Lett 74:644-650. doi: 10.1209/epl/i2005-10578-y

24. Scholtès L, Chareyre B, Nicot F, Darve F (2009) Micromechanics of granular materials with capillary effects. Int J Eng Sci 47:1460-1471. doi: 10.1016/j.ijengsci.2009.10.003

25. Golchert DJ, Moreno R, Ghadiri M, et al. (2004) Application of X-ray microtomography to numerical simulations of agglomerate breakage by distinct element method. Adv Powder Technol 15:447-457. doi: 10.1163/1568552041270554

26. Fu X, Dutt M, Bentham a. C, et al. (2006) Investigation of particle packing in model pharmaceutical powders using X-ray microtomography and discrete element method. Powder Technol 167:134-140. doi: 10.1016/j.powtec.2006.06.011

27. Moreno-Atanasio R, Williams RA, Jia X (2010) Combining X-ray microtomography with computer simulation for analysis of granular and porous materials. Particuology 8:81-99. doi: 10.1016/j.partic.2010.01.001

28. Wang Y-H, Leung S-C (2008) A particulate-scale investigation of cemented sand behavior. Can Geotech J 45:29-44. doi: 10.1139/T07-070

29. Wang YH, Leung SC (2008) Characterization of Cemented Sand by Experimental and Numerical Investigations. J Geotech Geoenvironmental Eng 134:992-1004.

30. Wang J-P, Li X, Yu H-S (2018) A micro - macro investigation of the capillary strengthening effect in wet granular materials . Acta Geotech. doi: doi.org/10.1007/s11440-017-0619-0 
31. Lame O, Bellet D, Di Michiel M, Bouvard D (2003) In situ microtomography investigation of metal powder compacts during sintering. Nucl Instruments Methods Phys Res Sect B Beam Interact with Mater Atoms 200:287-294. doi: 10.1016/S0168$583 X(02) 01690-7$

32. Williams R a., Jia X (2003) Tomographic imaging of particulate systems. Adv Powder Technol 14:1-16. doi: 10.1163/156855203762469867

33. Tengattini A, Andò E (2015) Kalisphera: an analytical tool to reproduce the partial volume effect of spheres imaged in 3D. Meas Sci Technol 26:095606. doi: $10.1088 / 0957-0233 / 26 / 9 / 095606$

34. Aste T, Saadatfar M, Senden T (2005) Geometrical structure of disordered sphere packings. Phys Rev E 71:1-15. doi: 10.1103/PhysRevE.71.061302

35. Al-Raoush R (2007) Microstructure characterization of granular materials. Phys A Stat Mech its Appl 377:545-558. doi: 10.1016/j.physa.2006.11.090

36. Farber L, Tardos G, Michaels JN (2003) Use of X-ray tomography to study the porosity 802 and morphology of granules. Powder Technol 132:57-63. doi: 10.1016/S0032a., Senden TJ (2004) Investigating the geometrical structure of disordered sphere packings. Phys A Stat Mech its Appl 339:16-23. doi: 10.1016/j.physa.2004.03.034

38. Aste T (2005) Variations around disordered close packing. J Phys Condens Matter

39. Andò E, Bésuelle P, Hall S a., et al. (2012) Experimental micromechanics: grain-scale observation of sand deformation. Géotechnique Lett 2:107-112. doi: 10.1680/geolett.12.00027

40. Khaddour G (2005) Multi-scale characterization of the hydro-mechanical behavior of unsaturated sand: water retention and triaxial responses. Université Grenoble Alpes 
814 41. Scheel M, Seemann R, Brinkmann M, et al. (2008) Morphological clues to wet granular 815 pile stability. Nat Mater 7:189.

816 42. Khaddour G, Riedel I, Andò E, et al. (2018) Grain-scale characterization of water 817 retention behaviour of sand using X-ray CT. Acta Geotech 13:497-512. doi: $818 \quad 10.1007 / \mathrm{s} 11440-018-0628-7$

819 43. Wang JP, Lambert P, De Kock T, et al. (2019) Investigation of the effect of specific 820 interfacial area on strength of unsaturated granular materials by X-ray tomography. Acta 821 Geotech. doi: 10.1007/s11440-019-00765-2

44. Moscariello M, Cuomo S, Salager S (2018) Capillary collapse of loose pyroclastic 823 unsaturated sands characterized at grain scale. Acta Geotech 13:117-133. doi: 10.1007/s11440-017-0603-8

45. Newitt DM, Conway-Jones JM (1958) A contribution to the theory and practice of granulation. Trans Inst Chem Eng 36:422.

827

46. Ridler TW, Calvard S (1978) Picture Thresholding Using an Iterative Slection Method. IEEE Trans Syst Man Cybern 8:630-632. doi: 10.1109/TSMC.1978.4310039

47. Xie L, Cianciolo RE, Hulette B, et al. (2012) Magnetic resonance histology of age-related nephropathy in the Sprague Dawley rat. Toxicol Pathol 40:764-78. doi: $10.1177 / 0192623312441408$

48. Peng T, Balijepalli A, Gupta SK, LeBrun T (2007) Algorithms for On-Line Monitoring of Micro Spheres in an Optical Tweezers-Based Assembly Cell. J Comput Inf Sci Eng

49. Illingworth J, Kittler J (1987) The Adaptive Hough Transform. IEEE Trans Pattern Anal Mach Intell PAMI-9:690-698. doi: 10.1109/TPAMI.1987.4767964

838 biocemented sand using 3D X-ray micro-tomography. Acta Geotech 14:597-613. doi: 
51. Lai Z, Chen Q (2019) Reconstructing granular particles from X-ray computed tomography using the TWS machine learning tool and the level set method. Acta Geotech. doi: $10.1007 / \mathrm{s} 11440-018-0759-\mathrm{x}$

52. Ding W, Howard AJ, Peri MDM, Cetinkaya C (2007) Rolling resistance moment of microspheres on surfaces: contact measurements. Philos Mag 87:5685-5696. doi:

53. Santamarina JC (2001) Soil Behavior at the Microscale: Particle Forces. In: Ladd CC (ed) Soil Behav. Soft Gr. Constr. pp 1-32

54. Torquato S (2002) Random Heterogeneous Materials: Microstructure and Macroscopic Properties. Springer-Verlag, New York

55. Donev A, Torquato S, Stillinger FH (2005) Pair correlation function characteristics of nearly jammed disordered and ordered hard-sphere packings. Phys Rev E - Stat

56. Agnolin I, Roux J-N (2007) Internal states of model isotropic granular packings. I. Assembling process, geometry, and contact networks. Phys Rev E 76:061302. doi: 10.1103/PhysRevE.76.061302

57. Bruchon J-F, Pereira J-M, Vandamme M, et al. (2013) X-ray microtomography powders: Influence of assembling procedure and contact laws on low consolidation

59. Tang A-M, Cui Y-J, Eslami J, Défossez P (2009) Analysing the form of the confined uniaxial compression curve of various soils. Geoderma 148:282-290. doi: 10.1016/j.geoderma.2008.10.012

60. Wiebicke M, Andò E, Herle I, Viggiani G (2017) On the metrology of interparticle 

contacts in sand from x-ray tomography images. Meas Sci Technol 28:124007.

867 61. Marmottant A, Salvo L, Martin CL, Mortensen A (2008) Coordination measurements in 868 compacted $\mathrm{NaCl}$ irregular powders using X-ray microtomography. J Eur Ceram Soc 869 28:2441-2449. doi: 10.1016/j.jeurceramsoc.2008.03.041

62. Aste T, Saadatfar M, Senden TJ (2006) Local and global relations between the number of 871 contacts and density in monodisperse sphere packs. J Stat Mech Theory Exp 872 2006:P07010. doi: 10.1088/1742-5468/2006/07/P07010

873 63. Al-Raoush RI, Willson CS (2005) Extraction of physically realistic pore network 874 properties from three-dimensional synchrotron X-ray microtomography images of 875 unconsolidated porous media systems. J Hydrol 300:44-64. doi: $876 \quad 10.1016 / j . j h y d r o l .2004 .05 .005$

877

878

879

880

881 
883

884 Figure 1: Scanning Electronic Microscopic image of a glass bead. 6

885 Figure 2: Void ratio versus vertical stress during oedometric compression tests for two

886 specimens. The circle points show the conditions of XRCT scans. .7

887 Figure 3: (a) Global scan, (b) local scan, and (c) investigated cube. 9

888 Figure 4: 3D reconstructed images of the sample at different compaction levels. (a) S1, (b) S2,

889 (c) S3, and (d) S4.

890 Figure 5. Grey level histogram for the four scans. 11

891 Figure 6: Void ratio versus elevation at different compaction levels. Note that the value in the 892 parenthesis is the average void ratio. 12

893 Figure 7: Algorithm used to detect the spherical structures. .14

894 Figure 8: Construction of the accumulation array from the gradient field: the nonzero gradient 895 vector added to the accumulation array (left), and the accumulation array after adding the nonzero gradient vectors in various directions (right).

897 Figure 9: Definition of the signature function. 16

898 Figure 10: An example of signature function computed from the 3D image. 17

899 Figure 11: A slice in a 3D detected image. 18

900 Figure 12: Method to find the lost contacts; the inner cube is the standard volume (SV) and

901 the outer one is the extended volume $(\mathrm{EV})$.

902 Figure 13: Example of reconstructed 3D specimens: (a, b) Original image - (c, d) Binary 903 images of the investigated cube; (e, f) Reconstructed 3D image with tolerance of 0 voxel; (g,

$904 \mathrm{~h})$ Reconstructed 3D image with tolerance of 1 voxel. .21

905 Figure 14: Solid fraction at different compaction levels. AD denotes after detection, BD 906 denotes before detection. 22 
907 Figure 15: Pore distance distribution for four compaction levels. In each level, gold region 908 denotes the values of ten cubes; continuous line denotes the average value...........................24

909 Figure 16: Average total coordination number for different compaction levels....................26

910 Figure 17: Average coordination numbers of close neighbors for different compaction levels.

911 .27

912 Figure 18: Average number of contacts per grain for different compaction levels. 28

913 Figure 19: An example of a sphere having one contact. 29

914 Figure 20: Radial distribution functions for particle centers of four compaction levels. 30

915 Figure 21: Contact network and reconstructed 3D image of a typical slice under growing of

916 compaction level. 33

917

918 